\title{
The Dirichlet Heat Kernel in Inner Uniform Domains in Fractal-Type Spaces
}

\author{
Janna Lierl ${ }^{1}$
}

Received: 20 January 2017 / Accepted: 24 January 2020 / Published online: 18 June 2021

(C) The Author(s) 2021, corrected publication 2022

\begin{abstract}
This paper proves two-sided estimates for the Dirichlet heat kernel on inner uniform domains in metric measure Dirichlet spaces satisfying the volume doubling condition, the Poincaré inequality, and a cutoff Sobolev inequality. More generally, we obtain local upper and lower bounds for the Dirichlet heat kernel on locally inner uniform domains under local geometric assumptions on the underlying space.
\end{abstract}

Keywords Dirichlet heat kernel · Fractals $\cdot h$-transform · Inner uniform domains · Ultracontractivity $\cdot$ Harnack inequality

Mathematics Subject Classification (2010) 35K08 $\cdot 31 \mathrm{C} 25 \cdot 60 \mathrm{~J} 35$

\section{Introduction}

This paper continues the study of the Dirichlet heat kernel by the Doob's transform technique. P. Gyrya and L. Saloff-Coste [20] proved that the Doob's $h$-transform of the Dirichlet heat kernel $p_{U}^{D}$ by a harmonic profile $h$ on an unbounded domain $U$ (i.e. $h$ is harmonic on $U$ and satisfies Dirichlet boundary condition),

$$
p_{h}(t, x, y):=\frac{p_{U}^{D}(t, x, y)}{h(x) h(y)},
$$

satisfies two-sided Gaussian bounds provided that $U$ is an inner uniform domain in a metric measure Dirichlet space that admits a global heat kernel satisfying Gaussian bounds. This yields two-sided bounds for the Dirichlet heat kernel in terms of the harmonic profile $h$,

$$
p_{U}^{D}(t, x, y) \asymp C \frac{h(x) h(y)}{\int_{B_{U}\left(x, \Psi^{-1}(t)\right)} h^{2} d \mu} \exp \left(-c \frac{d_{U}(x, y)^{2}}{t}\right),
$$

where $d_{U}$ is the intrinsic length metric of the domain $U$, and $\Psi(r)=r^{2}$ is the appropriate time-space scaling for a diffusion that shows classical Gaussian behavior.

Janna Lierl

ju14@cornell.edu

1 Institute of Mathematics, Technische Universität Clausthal, Erzstr. 1, 38678,

Clausthal-Zellerfeld, Germany 
Similarly, for the case of bounded inner uniform domains, L. Saloff-Coste and the author [27] obtained two-sided bounds for the Dirichlet heat kernel by proving Gaussian bounds for the kernel

$$
p_{\phi}(t, x, y):=\frac{p_{U}^{D}(t, x, y) e^{t \lambda}}{\phi(x) \phi(y)},
$$

where $\phi$ is the ground state and $\lambda$ is the lowest Dirichlet eigenvalue for the domain $U$. The Dirichlet heat kernel estimates obtained by this method imply the intrinsic ultracontractivity of the infinitesimal generator of the heat semigroup. In particular, they refine the estimates of, e.g., [5, 14] in the special case of inner uniform domains. In [27], Dirichlet heat kernels on locally inner uniform domains, and non-symmetric Dirichlet heat kernels were studied as well.

The aim of this paper is to prove two-sided estimates for Dirichlet heat kernels on bounded inner uniform domains and on locally inner uniform domains in fractal-type spaces such as the Sierpinski gasket where a global heat kernel exists and satisfies two-sided subGaussian estimates. The main results of this paper are stated in Section 3. As special cases, we recover the two-sided estimates for Dirichlet heat kernels of [20] and [27] (Gaussian case), and [22] (sub-Gaussian case). The setting of this paper is that of a symmetric strongly local regular Dirichlet space satisfying volume doubling, reverse volume doubling, Poincaré inquality and a cutoff Sobolev condition. Characterizations of sub-Gaussian heat kernel estimates by these conditions, or by the parabolic Harnack inequality, are given in, e.g., [10, 11, $18,19,21]$. The hypotheses on the underlying metric measure Dirichlet space are described in Section 1 where we also recall the definition of inner uniform domains and how it relates to the boundary Harnack principle. If we assume volume doubling and Poincaré inequality only locally, then we still get estimates for the Dirichlet heat kernel $p_{U}^{D}$ locally near two points $x$ and $y$ and for small time $t$. These local estimates hold on any locally inner uniform domain.

In Section 2, we consider Doob's $h$-transform and show that volume doubling, Poincaré inquality and the cutoff Sobolev inequality on annuli are preserved under Doob's $h$ transform.

In Section 4 we consider examples of inner uniform domains in the Sierpinski gasket. These are constructed by taking a line that cuts through the gasket as boundary of the domain. For horizontal or vertical lines, computations of harmonic functions and their boundary decay is known. We quote some of these examples. As can be seen from our main results, the boundary decay rate of the Dirichlet heat kernel at any fixed boundary point is the same as the boundary decay rate of the ground state $\phi$, which in turn is the same as the boundary decay rate of any function that is positive harmonic on some portion of the domain near that boundary point and vanishes along the boundary of the domain.

\section{Preliminaries}

\subsection{Basic setup}

Let $(X, d, \mu, \mathcal{E}, \mathcal{F})$ be a metric measure Dirichlet space. That is, $(X, d)$ is a locally compact separable metric space, $\mu$ is a positive Radon measure on $X$ with full support, and $(\mathcal{E}, \mathcal{F})$ is a symmetric strongly local regular Dirichlet form on $L^{2}(X, \mu)$. Throughout the paper we assume that $(X, d)$ is a length metric space. We let $(L, D(L))$ be the infinitesimal generator of $(\mathcal{E}, \mathcal{F})$. 
The Dirichlet form $(\mathcal{E}, \mathcal{F})$ admits an energy measure $d \Gamma(u, v)$, for each $u, v \in \mathcal{F}$. In particular, $\mathcal{E}(u, v)=\int_{X} d \Gamma(u, v)$. We will tacitly use the well-known properties of the energy measures such as chain rule and Cauchy-Schwarz type inequality; these are presented in [17, (3.2.27), Theorem 3.2.2, (3.2.28)] and [17, Lemma 3.2.5 and Lemma 5.6.1] where $\Gamma(u, u)$ is denoted as $\mu_{\langle u\rangle}$, and in [13].

For an open subset $U \subset X$, define

$\mathcal{F}_{\text {loc }}(U):=\left\{f \in L_{\text {loc }}^{2}(U, \mu): \forall\right.$ open rel. compact $A \subset U, \exists f^{\sharp} \in \mathcal{F},\left.f\right|_{A}=\left.f^{\sharp}\right|_{A} \mu$-a.e. $\}$,

where $L_{\text {loc }}^{2}(U, \mu)$ is the space of functions that are $L^{2}(\mu)$-integrable over any open relatively compact subset $A \subset U$. For $f, g \in \mathcal{F}_{\text {loc }}(U)$ we can define $\Gamma(f, g)$ locally by $\left.\Gamma(f, g)\right|_{A}:=$ $\left.\Gamma\left(f^{\sharp}, g^{\sharp}\right)\right|_{A}$, where $A \subset U$ is open relatively compact and $f^{\sharp}, g^{\sharp}$ are functions in $\mathcal{F}$ such that $f=f^{\sharp}, g=g^{\sharp} \mu$-a.e. on $A$. Indeed, this construction is justified by [13, Theorem 4.3.10(ii)] and relies on the fact that $\left.f\right|_{A}$ and $\left.f^{\sharp}\right|_{A}$ have quasi-continuous modifications $\tilde{f}$ and $\tilde{f}^{\sharp}$, respectively, which agree on $A$. Define

$$
\begin{aligned}
& \mathcal{F}(U):=\left\{u \in \mathcal{F}_{\text {loc }}(U): \int_{U}|u|^{2} d \mu+\int_{U} d \Gamma(u, u)<\infty\right\}, \\
& \mathcal{F}_{\mathrm{c}}(U):=\{u \in \mathcal{F}(U): \text { the essential support of } u \text { is compact in } U\}, \\
& \mathcal{F}^{0}(U):=\text { the closure of } \mathcal{F}_{\mathrm{c}}(U) \text { in }\left(\mathcal{F},\|\cdot\|_{\mathcal{F}}\right),
\end{aligned}
$$

where the $\mathcal{F}$-norm is defined as

$$
\|u\|_{\mathcal{F}}:=\left(\mathcal{E}(u, u)+\int_{X} u^{2} d \mu\right)^{1 / 2} .
$$

Note that $\mathcal{F}_{\mathrm{c}}(U)$ is a linear subspace of $\mathcal{F}$. We set

$$
\mathcal{E}_{U}^{D}(f, g):=\mathcal{E}(f, g), \quad f, g \in \mathcal{F}^{0}(U) .
$$

Notice that $\left(\mathcal{E}_{U}^{D}, \mathcal{F}^{0}(U)\right)$ is a Dirichlet form and the functions in its domain $\mathcal{F}^{0}(U)$ satisfy the Dirichlet boundary condition. Let $\left(L_{U}^{D}, D\left(L_{U}^{D}\right)\right)$ be the infinitesimal generator and $P_{U, t}^{D}, t>0$, be the semigroup associated with $\left(\mathcal{E}_{U}^{D}, \mathcal{F}^{0}(U)\right)$.

\subsection{Volume doubling, Poincaré and cutoff Sobolev inequalities}

In this section we recall geometric conditions that will be presumed in the main results. It is well known that these are satisfied on many self-similar fractals; we refer to [6-8, 10, 16, 23].

Let $Y \subset X$ be open and $R_{0} \in(0, \infty]$. We will assume that any ball $B(x, R)=\{y \in X$ : $d(x, y)<R\}$ with $R \in\left(0, R_{0}\right)$ is relatively compact whenever $B(x, 2 R) \subset Y$. In alignment with the notation in [27], we will call this condition A2- $Y$, or simply A2 when $Y=X$.

Note that A2- $Y$ holds whenever $\bar{Y}$ is complete in $(X, d)$, see, e.g., [33, Lemma 1.1(i)].

Definition 1.1 (i) The volume doubling property (VD) is satisfied on $Y$ up to scale $R_{0}$ if there exists a constant $C_{\mathrm{VD}} \in(0, \infty)$ such that for every ball $B(x, 2 R) \subset Y, R \in$ $\left(0, R_{0}\right)$,

$$
V(x, 2 R) \leq C_{\mathrm{VD}} V(x, R),
$$

where $V(x, R)=\mu(B(x, R))$ denotes the volume of the ball $B(x, R)$. 
(ii) The reverse volume doubling property (RVD) holds on $Y$ up to scale $R_{0}$ if there are constants $C_{\mathrm{RVD}}$ and $v_{0} \in[1, \infty)$ such that

$$
\frac{\mu(B(x, R))}{\mu(B(y, s))} \geq C_{\mathrm{RVD}}\left(\frac{R}{s}\right)^{v_{0}}
$$

for any ball $B(x, 2 R) \subset Y$ with $R \in\left(0, R_{0}\right)$ and $X \backslash B(x, R) \neq \emptyset$, and for all $s \in(0, R), y \in B(x, R)$.

Let $\Psi:[0, \infty) \rightarrow[0, \infty)$ be a continuous strictly increasing bijection which has the property that, for some constants $1<\beta_{1} \leq \beta_{2}<\infty$ and $C_{\Psi} \in[1, \infty)$,

$$
C_{\Psi}^{-1}\left(\frac{R}{r}\right)^{\beta_{1}} \leq \frac{\Psi(R)}{\Psi(r)} \leq C_{\Psi}\left(\frac{R}{r}\right)^{\beta_{2}}, \quad \forall 0<r \leq R<\infty .
$$

Definition $1.2(\mathcal{E}, \mathcal{F})$ satisfies the weak Poincaré inequality $\operatorname{PI}(\Psi)$ on $Y$ up to scale $R_{0}$ if there exists a constant $C_{\mathrm{PI}} \in(0, \infty)$ such that for any ball $B(x, 2 R) \subset Y$ with $R \in\left(0, R_{0}\right)$, and any $f \in \mathcal{F}$,

$$
\int_{B(x, R)}\left(f-f_{B}\right)^{2} d \mu \leq C_{\mathrm{PI}} \Psi(R) \int_{B(x, 2 R)} d \Gamma(f, f),
$$

where $f_{B}=\mu(B(x, R))^{-1} \int_{B(x, R)} f d \mu$.

Let $\mathcal{C}_{\mathrm{c}}(X)$ be the space of continuous functions with compact support in $X$.

Definition 1.3 Let $V$ and $U$ be open subsets of $X$ with $V \subset \bar{V} \subset U$. A function $\phi \in$ $\mathcal{F} \cap \mathcal{C}_{\mathrm{c}}(X)$ is called a cutoff function for $V$ in $U$ if $\phi$ takes values in $[0,1], \phi=1$ on $V$ and the compact support of $\phi$ is contained in $U$.

Definition 1.4 We say that $\operatorname{CSA}(\Psi)$ holds on $Y$ up to scale $R_{0}$ if there exists a constant $C_{0}>0$ such that for some $\epsilon \in(0,1)$, any $B(x, 2 R) \subset Y$ with $R \in\left(0, R_{0}\right)$, and any $r \in(0, R]$, there exists a cutoff function $\phi$ for $B(x, R)$ in $B(x, R+r)$ which satisfies

$$
\int_{A} f^{2} d \Gamma(\phi, \phi) \leq \epsilon \int_{A} \phi^{2} d \Gamma(f, f)+\frac{C_{0} \epsilon^{1-\beta_{2} / 2}}{\Psi(r)} \int_{A} f^{2} d \mu, \quad \forall f \in \mathcal{F},
$$

where $A=B(x, R+r) \backslash B(x, R)$.

If CSA $(\Psi)$ holds on $X$ up to scale $R_{0}=\infty$ for fixed $\epsilon=\frac{1}{8}$, then we simply say that $\mathrm{CSA}(\Psi)$ holds. This global condition was introduced in [4] and named the cutoff Sobolev inequality on annuli.

Remark 1.5 (i) If A2- $Y, \operatorname{RVD}$ and $\mathrm{CSA}(\Psi)$ hold on $Y$ up to scale $R_{0}$ for some $\epsilon \in(0,1)$, then it holds for all $\epsilon \in(0,1)$. See [24, Lemma 2.3].

(ii) If $\mathrm{A} 2-X, \mathrm{RVD}, \mathrm{VD}$ and $\mathrm{PI}(\Psi)$ hold on $X$ up to scale $R_{0}=\infty$, then $\operatorname{CSA}(\Psi)$ is equivalent to the generalized capacity condition introduced in [18]. In general, the generalized capacity condition is weaker than $\operatorname{CSA}(\Psi)$ since it allows the cutoff function $\phi$ to depend on the function $f$.

We will drop the words "up to scale $R_{0}$ " in any of the above conditions when $R_{0}=\infty$. 
We say that $\mathrm{A} 2, \mathrm{VD}, \mathrm{RVD}$, or $\mathrm{PI}(\Psi)$ holds locally on $X$ if every point $x \in X$ has a neighborhood $Y_{x}=B\left(x, 4 r_{x}\right)$ where A2- $Y_{x}, \mathrm{VD}, \mathrm{RVD}$, or PI $(\Psi)$, respectively, holds up to scale $R_{0}=4 r_{x} \leq \frac{1}{2} \operatorname{diam}(X)$. Here, $\operatorname{diam}(X)$ denotes the diameter of $(X, d)$.

\subsection{Harnack Inequalities}

Let $I$ be an open time interval and $U \subset X$ an open. For the definition of a local weak solution to the heat equation on $Q=I \times U$, we will use the following function spaces. Recall that $\mathcal{F}$ is the domain of the Dirichlet form $(\mathcal{E}, \mathcal{F})$. We denote by $\mathcal{F}^{\prime}$ the dual space of $\mathcal{F}$. Let $L^{2}(I \rightarrow \mathcal{F})$ be the Hilbert space of those functions $v: I \rightarrow \mathcal{F}$ that have finite norm

$$
\|v\|_{L^{2}(I \rightarrow \mathcal{F})}:=\left(\int_{I}\|v(t)\|_{\mathcal{F}}^{2} d t\right)^{1 / 2} .
$$

Similarly, we define the space $L^{2}\left(I \rightarrow \mathcal{F}^{\prime}\right)$. Observe that we can identify $L^{2}\left(I \rightarrow \mathcal{F}^{\prime}\right)$ with the dual space of $L^{2}(I \rightarrow \mathcal{F})$, and we may write $v(t, x)$ for $(v(t))(x)$.

Let $W^{1}\left(I \rightarrow \mathcal{F}^{\prime}\right) \subset L^{2}\left(I \rightarrow \mathcal{F}^{\prime}\right)$ be the Hilbert space of those functions $v: I \rightarrow \mathcal{F}^{\prime}$ who have a distributional time-derivative that can be represented by a function in $L^{2}(I \rightarrow$ $\left.\mathcal{F}^{\prime}\right)$. Identifying $L^{2}\left(I \rightarrow L^{2}(X)\right)$ with its dual space, we set

$$
\mathcal{F}(Q):=L^{2}(I \rightarrow \mathcal{F}) \cap W^{1}\left(I \rightarrow \mathcal{F}^{\prime}\right) .
$$

Let $\mathcal{F}_{\text {loc }}(Q)$ be the space of all measurable functions $u: Q \rightarrow \mathbb{R}$ such that for any open interval $J$ relatively compact in $I$, and any open subset $A$ relatively compact in $U$, there exists a function $u^{\sharp} \in \mathcal{F}(I \times X)$ such that $u^{\sharp}=u$ a.e. in $J \times A$.

Definition 1.6 A function $u: Q \rightarrow \mathbb{R}$ is a local weak solution of the heat equation $\frac{\partial}{\partial t} u=$ $L u$ in $Q$, if

(i) $u \in \mathcal{F}_{\text {loc }}(Q)$,

(ii) for any $\phi \in \mathcal{F}_{\mathrm{c}}(U), \int_{I}\left\langle\frac{\partial}{\partial t} u(t, \cdot), \phi(t, \cdot)\right\rangle_{\mathcal{F}^{\prime}, \mathcal{F}} d t+\int_{I} \mathcal{E}(u(t, \cdot), \phi) d t=0$.

For other notions of a local weak solution which are equivalent to the one given above see, e.g., [26] and references therein. The existence and uniqueness of weak solutions is known from PDE literature [29, Section 4 in Chapter 3] and [35].

We say that $(\mathcal{E}, \mathcal{F})$ satisfies the (strong) parabolic Harnack inequality $\mathrm{PHI}(\Psi)$ on $Y$ up to scale $R_{0}$ if, for any parameters $\delta \in(0,1)$ and $0<\tau_{1}<\tau_{2}<\tau_{3}<\tau_{4} \leq 1$, there is a constant $C_{\mathrm{PHI}} \in(0, \infty)$ such that for any $a \in \mathbb{R}$, any ball $B(x, 4 r) \subsetneq B(x, 8 r) \subset Y$, $0<r<\frac{R_{0}}{4}$, and any non-negative local weak solution $u$ of the heat equation for $L$ in $Q=Q(x, a, r)$, we have

$$
\sup _{Q^{-}} u \leq C_{\mathrm{PHI}} \inf _{Q^{+}} u,
$$

where $B=B(x, r), \delta B=B(x, \delta r)$,

$$
\begin{aligned}
Q & =Q(x, a, r)=(a, a+\Psi(r)) \times B, \\
Q^{-} & =\left(a+\tau_{1} \Psi(r), a+\tau_{2} \Psi(r)\right) \times \delta B, \\
Q^{+} & =\left(a+\tau_{3} \Psi(r), a+\tau_{4} \Psi(r)\right) \times \delta B .
\end{aligned}
$$

Definition 1.7 Let $V \subset X$ be open and $\lambda \in \mathbb{R}$. A function $u: V \rightarrow \mathbb{R}$ is $\mathcal{E}-\lambda$-harmonic on $V$ if

(i) $u \in \mathcal{F}_{\text {loc }}(V)$, 
(ii) For any function $\phi \in \mathcal{F}_{\mathrm{c}}(V)$,

$$
\mathcal{E}(u, \phi)-\lambda \int u \phi d \mu=0 .
$$

If $\lambda=0$, we simply say that $u$ is harmonic or $\mathcal{E}$-harmonic.

Harmonic functions are time-independent weak solutions of the heat equation. Therefore, the following elliptic Harnack inequality is an immediate consequence of the parabolic Harnack inequality $\mathrm{PHI}(\Psi)$.

The Dirichlet form $(\mathcal{E}, \mathcal{F})$ satisfies the elliptic Harnack inequality (EHI) on $Y$ up to scale $R_{0}$ if for any $\delta \in(0,1)$ there is a constant $C_{\mathrm{EHI}}>0$ such that, for any ball $B(x, 2 r) \subsetneq B(x, 4 r) \subset Y$ with $r \in\left(0, R_{0} / 4\right)$, and any function $u \in \mathcal{F}$ that is non-negative and harmonic on $B(x, r)$, we have

$$
\sup _{B(x, \delta r)} u \leq C_{\mathrm{EHI}} \inf _{B(x, \delta r)} u .
$$

Let $U$ be an open subset of $X$. Define

$$
\begin{aligned}
\mathcal{F}_{\text {loc }}^{0}(U):= & \left\{f \in L_{\text {loc }}^{2}(U, \mu): \forall \text { open } A \subset U \text { rel. compact in } \bar{U}\right. \text { there exists } \\
& \left.f^{\sharp} \in \mathcal{F}^{0}(U): f^{\sharp}=f \mu \text {-a.e. on } A\right\} .
\end{aligned}
$$

If $U$ is connected, then $U$ admits an inner metric

$$
d_{U}(x, y):=\inf \{\operatorname{length}(\gamma) \mid \gamma:[0,1] \rightarrow U \text { continuous, } \gamma(0)=x, \gamma(1)=y\},
$$

where

$$
\text { length }(\gamma):=\sup \left\{\sum_{i=1}^{n} d\left(\gamma\left(t_{i}\right), \gamma\left(t_{i-1}\right)\right): n \in \mathbb{N}, 0 \leq t_{0}<\ldots<t_{n} \leq 1\right\} .
$$

The inner metric $d_{U}$ is indeed finite because $U$ is connected and $(X, d)$ is a length metric space by assumption. Let $\widetilde{U}$ be the completion of $U$ with respect to $d_{U}$, and let $\partial_{\widetilde{U}} U=\widetilde{U} \backslash U$ be the boundary. For $x \in \widetilde{U}$, we will consider the inner balls $B_{\widetilde{U}}(x, r):=\{y \in \widetilde{U}$ : $\left.d_{U}(x, y)<r\right\}$ and $B_{U}(x, r):=B_{\widetilde{U}}(x, r) \cap U$.

For a set $V \subset U$ let $\operatorname{diam}_{U}(V):=\sup _{x, y \in V} d_{U}(x, y)$ be the diameter of $V$ with respect to the inner metric $d_{U}$. For an open subset $V \subset U$, define

$$
\begin{aligned}
& \mathcal{F}_{\text {loc }}^{0}(U, V):=\left\{f \in L_{\text {loc }}^{2}(V, \mu): \forall \text { open } A \subset V \text { rel. compact in } \bar{U}\right. \text { with } \\
&\left.d_{U}(A, U \backslash V)>0, \exists f^{\sharp} \in \mathcal{F}^{0}(U): f^{\sharp}=f \mu \text {-a.e. on } A\right\} .
\end{aligned}
$$

Note that $\mathcal{F}_{\text {loc }}^{0}(U)$ is the same as $\mathcal{F}_{\text {loc }}^{0}(U, U)$.

Definition 1.8 Let $V \subset U$ be open. We say that a harmonic function $u: V \rightarrow \mathbb{R}$ satisfies Dirichlet boundary condition along $\partial U$ if $u \in \mathcal{F}_{\text {loc }}^{0}(U, V)$.

Definition 1.9 Let $c \in(0,1), C \in(1, \infty)$. A $(c, C)$-inner uniform path in $U$ is a curve $\gamma:[\alpha, \beta] \rightarrow U$ such that

$$
d_{U}(\gamma(t), \tilde{U} \backslash U) \geq c \cdot \min \left\{d_{U}(\gamma(\alpha), \gamma(t)), d_{U}(\gamma(t), \gamma(\beta))\right\}, \quad \forall t \in[\alpha, \beta],
$$

and

$$
\text { length }(\gamma) \leq C \cdot d_{U}(\gamma(\alpha), \gamma(\beta)) \text {. }
$$

The domain $U$ is called a $(c, C)$-inner uniform domain if any two points in $U$ can be joined by a $(c, C)$-inner uniform path in $U$. 
Fix $c \in(0,1)$ and $C \in(1, \infty)$. For $\xi \in \partial_{\widetilde{U}} U$, let $R_{\xi}$ be the largest radius so that

(i) $B\left(\xi, 5(24 C+8 / c+35) R_{\xi}\right) \subsetneq X$

(ii) $\frac{8}{c} R_{\xi}<\frac{1}{2} \operatorname{diam}_{U}(U)$, if $\operatorname{diam}_{U}(U)<\infty$,

(iii) Any two points in $B_{U}\left(\xi, 2\left(24 C+\frac{4}{c}+31\right) R_{\xi}\right)$ can be connected by a curve that is $(c, C)$-inner uniform in $U$.

Let $W$ be a domain in $U$. Let $W^{\sharp}$ be the largest open set in $\widetilde{U}$ whose intersection with $U$ is $W$.

Definition 1.10 We say that $U$ is locally $(c, C)$-inner uniform up to scale $R_{0}$ near $W$, if $R_{\xi}>R_{0}$ for all $\xi \in W^{\sharp}$.

The metric space $(X, d)$ is geodesic if for any $x, y \in X$ there is a continuous map $\gamma:[0,1] \rightarrow X$ such that $\gamma(0)=x, \gamma(1)=y$ and $d(\gamma(s), \gamma(t))=|s-t| d(x, y)$ for all $s, t \in[0,1]$.

Let $C_{0}$ be the space of continuous functions $f: X \rightarrow \mathbb{R}$ that vanish at infinity.

Proposition 1.11 Suppose $(X, d)$ is geodesic, $A 2, C S A(\Psi)$ hold globally on $X$, and VD, $P I(\Psi)$ hold locally on $X$. Then the Markovian transition function $\left(\mathcal{P}_{t}\right)_{t>0}$ associated with $(\mathcal{E}, \mathcal{F})$ has the Feller-type property

$$
\mathcal{P}_{t} C_{0} \subset C_{0}, \forall t \geq 0, \quad \text { and } \quad \lim _{t \rightarrow 0}\left\|\mathcal{P}_{t} f-f\right\|_{\infty}=0, \forall f \in C_{0} \text {, }
$$

and the strong Feller property

for any bounded Borel measurable function $f: X \rightarrow \mathbb{R}, \mathcal{P}_{t} f$ is continuous.

Proof The strong Feller property is proved in [25, Proposition 3.2] under the stronger assumption that $(X, d)$ is complete and geodesic, VD holds, and weak heat kernel estimates $\operatorname{HKE}(\Psi)$ hold. However, the same proof goes through under the present weaker assumptions. Indeed, all that is needed for the proof of the strong Feller property is the existence and joint continuity of the heat kernel and a heat kernel upper bound. These are proved [24, Section 6]. Similarly, we can show that the Markovian transition functions $\mathcal{P}_{t}$ map the space $\mathcal{C}_{0}$ of continuous functions vanishing at infinity into itself, by repeating the proof given in [25, Proposition 3.2] under the present weaker assumptions and by applying the results of [24, Section 6].

It remains to show that $\lim _{t \rightarrow 0}\left\|\mathcal{P}_{t} f-f\right\|_{\infty}=0$ for any $f \in \mathcal{C}_{0}$. The proof in [25, Proposition 3.2] uses a conservativeness argument which does not apply here. However, the assertion can be proved by approximating $\mathcal{P}_{t} f$ by $\mathcal{P}_{t} g$ for some continuous $g$. Choosing $g$ to be constant on a neighborhood $U$ of an arbitrary point $x_{0} \in X$, it is possible to extend $\mathcal{P}_{t} g$ to a local weak solution on the whole time interval $(-\infty, \infty)$ by setting it equal to $g\left(x_{0}\right)$ on $(-\infty, 0] \times U$. The details of this proof are as in [15, Proof of Assumption 4 on page 1187].

Theorem 1.12 (Boundary Harnack principle) Suppose $(X, d)$ is geodesic, and $A 2, V D$, $P I(\Psi), C S A(\Psi)$ hold locally on $X$. Let $U$ be a domain in $X$. Then there are constants $A_{0}, A_{1}, A_{2}, A_{3} \in(1, \infty)$ such that the following holds. Let $\xi \in \partial_{\widetilde{U}} U$ with $R_{\xi}>0$. Let $r \in\left(0, \frac{8}{9} R_{\xi}\right)$ be small enough so that $A 2, V D, P I(\Psi)$ and $C S A(\Psi)$ hold on $Y=B\left(\xi, A_{2} r\right)$ 
up to scale $R_{0} \geq A_{3} r$. Let $u$, $v$ be two non-negative functions that are harmonic on $B_{U}\left(\xi, A_{0} r\right)$ with Dirichlet boundary condition along $\partial U$. Then

$$
\frac{u(x)}{u\left(x^{\prime}\right)} \leq A_{1} \frac{v(x)}{v\left(x^{\prime}\right)}
$$

for all $x, x^{\prime} \in B_{U}(\xi, r)$. The constants $A_{0}, A_{1}, A_{2}, A_{3}$ are independent of $U, \xi, r, u, v$, but may depend on the inner uniformity constants $c, C$, on $\beta_{1}, \beta_{2}, C_{\Psi}$, and on the constants in $V D, \operatorname{PI}(\Psi), C S A(\Psi)$.

Proof The relation between the inner uniformity of a domain in Euclidean space and the boundary Harnack principle has been studied in $[1,2]$. The boundary Harnack principle is proved in [25, Theorem 5.2] under the hypotheses that $(X, d)$ is geodesic, and that VD and weak heat kernel estimates w-HKE $(\Psi)$ hold globally on $X$. These global hypotheses are used to prove the Feller property of $(\mathcal{E}, \mathcal{F})$, the Poincare inequality, resistance estimates, elliptic Harnack inequality and certain bounds for the Dirichlet heat kernel on balls.

Under the weaker assumptions of Theorem 1.12, the resistance estimate $\operatorname{RES}(\Psi)$ can still be proved as in [9, Lemma 5.1]. The Feller property is already proved in Proposition 1.11 (replacing $(\mathcal{E}, \mathcal{F})$ by $(\mathcal{E}, \mathcal{F}(Y))$, we may assume that $\mathrm{A} 2$ and $\operatorname{CSA}(\Psi)$ hold globally). The elliptic Harnack inequality EHI (on $Y$ up to scale $R_{0}$ ) is a consequence of the parabolic Harnack inequality $\mathrm{PHI}(\Psi)$ proved in $[24$, Theorem 5.3] under A2- $Y, \mathrm{VD}, \mathrm{PI}(\Psi)$ and $\mathrm{CSA}(\Psi)$ (on $Y$ up to scale $R_{0}$ ). The existence and joint continuity of the heat kernel, and sufficient upper and lower bounds for the heat kernel and for Dirichlet heat kernels are proved in [24, Section 6].

Thus, the reasoning of [25, Section 3.2, Section 4, Section 5] goes through under the weaker assumptions of Theorem 1.12, proving the boundary Harnack principle.

We need the following two known results for the proof of our main results, and restate them here for the convenience of the reader.

Lemma 1.13 Let $x \in U$ and $r>0$ such that $B_{U}(x, r)$ has minimal radius (i.e. $B_{U}(x, r) \neq$ $B_{U}(x, s)$ for all $\left.s<r\right)$. Suppose that any two points in $B_{U}(x, r)$ can be connected by a path that is $(c, C)$-inner uniform in $U$. Then there exists a point $x_{r} \in B_{U}(x, r)$ with $d_{U}\left(x, x_{r}\right)=r / 4$ and $d_{U}\left(x_{r}, \widetilde{U} \backslash U\right) \geq c r / 8$.

The proof of this lemma is given in [20, Lemma 3.20].

Corollary 1.14 (Carleson estimate) Suppose $(X, d)$ is geodesic, and A2, VD, PI $(\Psi)$, $C S A(\Psi)$ hold locally on $X$. Then there are constants $A_{0}, A_{1}, A_{2}, A_{3} \in(1, \infty)$ such that the following holds. Let $\xi \in \partial_{\widetilde{U}} U$ with $R_{\xi}>0$. Let $r \in\left(0, \frac{1}{3} R_{\xi}\right)$ be small enough so that $A 2$, $V D, P I(\Psi)$ and $C S A(\Psi)$ hold on $Y=B\left(\xi, A_{2} r\right)$ up to scale $R_{0} \geq A_{3} r$. Let $u$ be a nonnegative harmonic function on $B_{U}\left(\xi, A_{0} r\right)$ with Dirichlet boundary condition along $\partial U$. Then

$$
u(y) \leq A_{1} u\left(x_{r}\right) \quad \forall x, y \in B_{U}(x, r / 2),
$$

where $x_{r}$ is as in Lemma 1.13. The constants $A_{0}, A_{1}, A_{2}, A_{3}$ are independent of $\xi, r, u$, but may depend on the inner uniformity constants $c, C$, on $\beta_{1}, \beta_{2}, C_{\Psi}$, and on the constants in $V D, \operatorname{PI}(\Psi), C S A(\Psi)$.

The Carleson estimate follows from the boundary Harnack principle of Theorem 1.12, and the proof is as in [25, Proof of Corollary 1] and [20, Theorem 4.17].

Let $p: \widetilde{U} \rightarrow \bar{U}$ be the natural projection into the closure $\bar{U}$ of $U$ in $(X, d)$, namely the unique continuous map such that $\left.p\right|_{U}$ is the identity map on $U$. For $x \in \widetilde{U}$ and $D=$ 
$B(p(x), r)$, let $D^{\prime}$ be the unique connected component of $p^{-1}(D \cap \bar{U})$ that contains $x$. We identify the subset $D^{\prime} \cap p^{-1}(U)$ of $\left(\widetilde{U}, d_{U}\right)$ with the subset $p\left(D^{\prime}\right) \cap U$ of $(X, d)$ and simply denote it by $D^{\prime} \cap U$. It follows that $D^{\prime} \cap U$ is the connected component of $D \cap U$ whose closure in $\widetilde{U}$ contains $x$.

The proof of the next lemma is given in [28, Lemma 3.7, Remark 3.8(ii)].

Lemma 1.15 Let $U$ be a domain in $X$. Let $x \in \widetilde{U}$ and $0<r<\frac{1}{2} \operatorname{diam}(X)$. Suppose that any two points in $B_{U}(x, r)$ can be connected by a path that is $(c, C)$-inner uniform in $U$. Suppose that $A 2-Y$ and $V D$ hold on $Y=B(p(x), 4 r) \subset X$ up to scale $R_{0}=4 r$. Then there exists a constant $C_{U} \in[1, \infty)$ such that for $D=B\left(p(x), r / C_{U}\right)$, we have

$$
B_{\widetilde{U}}\left(x, r / C_{U}\right) \subset D^{\prime} \subset B_{\widetilde{U}}(x, r) .
$$

The constant $C_{U}$ depends only on the volume doubling constant $C_{V D}$ and on the inner uniformity constants $c, C$. It does not depend on $x$ or $r$.

\section{Doob's $h$-transform Technique}

For this section, we let $(X, d, \mu, \mathcal{E}, \mathcal{F})$ be a metric measure Dirichlet space as in Section 1.1. In addition, we assume that $d$ is a length metric and A2 holds. We fix a domain $U \subset X$ and a subdomain $W \subset U$. Let $R_{0} \in(0, \infty]$.

\subsection{Doob's $h$-transform}

Definition 2.1 For a positive continuous function $h$ on $W$, the Doob's $h$-transform of $\left(\mathcal{E}_{W}^{D}, \mathcal{F}^{0}(W)\right)$ is the bilinear form $\left(\mathcal{E}^{h}, \mathcal{F}^{h}\right)$ defined as the closure of

$$
\mathcal{E}^{h}(f, g):=\int_{W} h^{2} d \Gamma(f, g), \quad f, g \in \mathcal{F}_{\mathrm{c}}(W),
$$

for the norm

$$
\|f\|_{\mathcal{F}^{h}}:=\left(\int_{W} h^{2} d \Gamma(f, f)+\int_{W} f^{2} h^{2} d \mu\right)^{1 / 2} .
$$

It follows easily from the definition that $\left(\mathcal{E}^{h}, \mathcal{F}^{h}\right)$ is a symmetric strongly local regular Dirichlet form on $L^{2}\left(W, h^{2} d \mu\right)$. We will write $\mathcal{F}_{\mathrm{b}}^{h}:=\mathcal{F}^{h} \cap L^{\infty}\left(W^{\sharp}, h^{2} d \mu\right)$ and $\mathcal{F}_{\mathrm{c}}^{h}:=$ $\left\{f \in \mathcal{F}^{h}: f\right.$ has compact support in $\left.W^{\sharp}\right\}$.

For a positive continuous function $h$ on $W$, let

$$
H: L^{2}\left(W, h^{2} d \mu\right) \rightarrow L^{2}(W, \mu), \quad f \mapsto h f .
$$

Then the linear subspace

$$
H^{-1}\left(\mathcal{F}^{0}(W)\right):=\left\{f / h: f \in \mathcal{F}^{0}(W)\right\}
$$

is dense in $L^{2}\left(W, h^{2} d \mu\right)$ and a Hilbert space with inner product

$$
<f, g>_{H^{-1}\left(\mathcal{F}^{0}(W)\right)}:=\mathcal{E}_{W}^{D}(h f, h g)+\int f g h^{2} d \mu .
$$

The following lemma is proved in [27, Lemma 6.5]. See also [20, Proof of Proposition 5.7]. 
Lemma 2.2 If $h$ is a positive continuous function in $\mathcal{F}_{\text {loc }}(W)$, then

$$
H^{-1}\left(\mathcal{F}_{c}(W) \cap L^{\infty}(W, \mu)\right)=\mathcal{F}_{c}(W) \cap L^{\infty}(W, \mu)=\mathcal{F}_{c}(W) \cap L^{\infty}\left(W, h^{2} d \mu\right),
$$

and this space is dense in the Hilbert space $H^{-1}\left(\mathcal{F}^{0}(W)\right)$. In particular, $\mathcal{F}_{c}(W) \cap$ $L^{\infty}(W, \mu)$ is a dense subspace of both $H^{-1}\left(\mathcal{F}^{0}(W)\right)$ and $\mathcal{F}^{h}$.

Lemma 2.3 Let $V$ be a non-empty open set in $U$. A bounded function $f$ defined on $V$ belongs to $\mathcal{F}_{\mathrm{loc}}^{0}(U, V)$ if and only if we have $\left.\psi\right|_{V} f \in \mathcal{F}^{0}(V)$ for any continuous function $\psi \in \mathcal{F}$ so that $\left.\psi\right|_{V}$ has compact support in $V^{\sharp}$ and $\left.\psi\right|_{V}$ extends continuously to $V^{\sharp}$.

Proof We follow the line of reasoning in [20, Proof of Lemma 2.36].

We first prove the forward implication. Let $\psi \in \mathcal{F}$ so that $\left.\psi\right|_{V}$ has compact support in $V^{\sharp}$ and $\left.\psi\right|_{V}$ is continuous and extends continuously to $V^{\sharp}$. For a bounded function $f \in$ $\mathcal{F}_{\text {loc }}^{0}(U, V)$ and any compact set $K \subset V^{\sharp}$, there exists a bounded function $f^{\sharp} \in \mathcal{F}^{0}(V)$ so that $f=f^{\sharp} \mu$-a.e. on $K \cap V$. Let $\left(f_{n}\right) \subset \mathcal{C}_{\mathrm{c}}(V) \cap \mathcal{F}^{0}(V)$ be a sequence that converges in $\mathcal{F}^{0}(V)$ to $f^{\sharp}$, and $\left|f_{n}\right| \leq M$ for all $n$, for some $M \in(0, \infty)$. Passing to a subsequence, we may assume that $f_{n} \rightarrow f$ quasi-everywhere, by [17, Theorem 2.1.4(i)]. Then $\psi f_{n} \in \mathcal{F}_{\mathrm{c}}(V)$ and

$$
\int d \Gamma\left(\psi f_{n}, \psi f_{n}\right) \leq 2 \int \psi^{2} d \Gamma\left(f_{n}, f_{n}\right)+2 \int f_{n}^{2} d \Gamma(\psi, \psi) .
$$

By the dominated convergence theorem, we obtain that $\left(\psi f_{n}\right)$ is a Cauchy sequence in $\mathcal{F}^{0}(V)$. Hence, $\left(\psi f_{n}\right)$ converges to $\left.\psi\right|_{V} f \in \mathcal{F}^{0}(V)$ by the closedness of $(\mathcal{E}, \mathcal{F})$.

To prove the converse, let $f$ be a bounded function on $V$. Let $K$ be a compact set in $V^{\sharp}$. Take a continuous function $\psi \in \mathcal{F}$ that takes constant value 1 on $K \cap V$ and so that $\left.\psi\right|_{V}$ has compact support in $V^{\sharp}$. As $\left.\psi\right|_{V} f(x)=f(x)$ for $\mu$-a.e. $x \in K \cap V$, if we know that $\left.\psi\right|_{V} f$ is in $\mathcal{F}^{0}(V)$ (for an arbitrary compact set $K \subset V^{\sharp}$ ), then we must have $f \in \mathcal{F}_{\text {loc }}^{0}(U, V)$.

A proof of the following proposition in the special case when $U=W$ is an unbounded inner uniform domain and $R_{0}=\infty$ will be given in [22].

Proposition 2.4 For a positive continuous function $h \in \mathcal{F}_{\text {loc }}(W)$, the Dirichlet form $\left(\mathcal{E}^{h}, \mathcal{F}^{h}\right)$ is regular on $L^{2}\left(W^{\sharp}, h^{2} d \mu\right)$.

Proof As $\left(\mathcal{E}^{h}, \mathcal{F}^{h}\right)$ is regular on $L^{2}\left(W, h^{2} d \mu\right)$, the space $\mathcal{C}_{\mathrm{c}}(W) \cap \mathcal{F}^{h}$ is dense in $\mathcal{F}^{h}$. We show that $\mathcal{C}_{\mathrm{c}}\left(W^{\sharp}\right) \cap \mathcal{F}^{h}$ is dense in $\mathcal{C}_{\mathrm{c}}\left(W^{\sharp}\right)$ with respect to uniform convergence. Pick any $u \in \mathcal{C}_{\mathrm{c}}\left(W^{\sharp}\right)$ with compact support $K \subset W^{\sharp}$. Consider the collection $\mathcal{C}_{K, n}$ of functions in $\mathcal{C}_{\mathrm{c}}\left(W^{\sharp}\right) \cap \mathcal{F}^{h}$ whose absolute value is at most $\frac{1}{n}$ outside $K$. Restricting the functions in $\mathcal{C}_{K, n}$ to $K$, we obtain a collection of functions in $\mathcal{C}(K)$ which separates the points of $K$ and contains the function that takes constant value $\frac{1}{n}$ on $K$; this will be proved below.

By the Stone-Weierstrass theorem, e.g. [34, Theorem 44.5], there exists a sequence $\left(f_{n, m}\right)_{m} \subseteq C_{K, n}$ such that $\sup _{K}\left|f_{n, m}-u\right|<\frac{1}{m}$. The diagonal sequence $\left(f_{n, n}\right)_{n}$ converges to $u$ uniformly on $W^{\sharp}$.

It remains to verify the required properties of $\mathcal{C}_{K, n}$.

We first show that there exists a function in $\mathcal{C}_{K, n}$ which takes constant value $\frac{1}{n}$ on $K$. Indeed, since $(\mathcal{E}, \mathcal{F})$ is regular, there exists a continuous function $\phi \in \mathcal{F}$ which takes value $\frac{1}{n}$ on an open neighborhood of $p(K)$, and so that $\left.\phi\right|_{W}$ has compact support in $W^{\sharp}$. Then $\left.\phi\right|_{W} h \in \mathcal{F}^{0}(W)$ by Lemma 2.3 , consequently $\left.\phi\right|_{W} \in H^{-1}\left(\mathcal{F}^{0}(W)\right)$. By Lemma 2.2 , there exists a sequence $\left(\phi_{n}\right) \subset \mathcal{F}_{\mathrm{c}}(W) \cap L^{\infty}\left(W, h^{2} d \mu\right)$ such that $\left.h \phi_{n} \rightarrow h \phi\right|_{W}$ in 
$\mathcal{F}^{0}(W)$. We may assume that $\left(h \phi_{n}\right)$ are uniformly bounded. Passing to a subsequence, we may assume that $h \phi_{n}$ converges to $\left.h \phi\right|_{W}$ quasi-everywhere. In particular, $\phi_{n}$ converges to $\left.\phi\right|_{W}$ quasi-everywhere. By the Leibniz rule and Cauchy-Schwarz inequality,

$$
\begin{aligned}
\int h^{2} d \Gamma\left(\phi_{n}, \phi_{n}\right) & =\int h d \Gamma\left(h \phi_{n}, \phi_{n}\right)-\int h \phi_{n} d \Gamma\left(h, \phi_{n}\right) \\
& \leq\left(\int h^{2} d \Gamma\left(\phi_{n}, \phi_{n}\right)\right)^{1 / 2}\left[\left(\int d \Gamma\left(h \phi_{n}, h \phi_{n}\right)\right)^{1 / 2}+\left(\int \phi_{n}^{2} d \Gamma(h, h)\right)^{1 / 2}\right] .
\end{aligned}
$$

Squaring each side and rearranging,

$$
\int h^{2} d \Gamma\left(\phi_{n}, \phi_{n}\right) \leq 2 \int d \Gamma\left(h \phi_{n}, h \phi_{n}\right)+2 \int \phi_{n}^{2} d \Gamma(h, h) .
$$

As explained above, the right hand side converges as $n \rightarrow \infty$. Passing again to a subsequence, this shows that the subsequence $\left(\phi_{n}\right)$ converges in $\mathcal{F}^{h}$, and its limit must be $\left.\phi\right|_{W}$. In particular, $\left.\phi\right|_{W} \in \mathcal{F}^{h}$. By construction, $\left.\phi\right|_{W}$ extends continuously to $W^{\sharp}$.

Next, we show that $\mathcal{C}_{K, n}$ separates any two distinct points $x, y \in K$. This property clearly holds if at least one of the points is in $K \cap W$. For two distinct points $x, y \in K \backslash W$, their inner distance $d_{U}(x, y)$ is positive. Let $0<d<d_{U}(x, y) / 2$ small. By regularity of $(\mathcal{E}, \mathcal{F})$, we can find a function $f \in \mathcal{C}_{\mathrm{c}}(X) \cap \mathcal{F}$ so that $f(p(x))=1$ and $f=0$ outside $B(p(x), d)$. Setting $\phi(z)=f(p(z))$ if $z$ is in the same connected component of $p^{-1}(B(p(x), d))$ as $x$, and $\phi(z)=0$ otherwise, we obtain that $\phi(x)=1$ and $\phi(y)=0$. When $d$ is small enough, $\phi$ is in $\mathcal{C}_{\mathrm{c}}\left(W^{\sharp}\right)$. We have $\left.\phi\right|_{W} h \in \mathcal{F}^{0}(W)$ by Lemma 2.3. By the same reasoning as above, it follows that $\left.\phi\right|_{W} \in \mathcal{F}^{h}$.

\subsection{Harmonic profiles}

Let $W \subset U \subset X$ be as in the previous subsection and $\lambda \in \mathbb{R}$.

Definition 2.5 (i) An $\mathcal{E}-\lambda$-harmonic profile in $U$ is a function $h$ on $U$ such that

(a) $h$ is $\mathcal{E}-\lambda$-harmonic in $U$,

(b) $h \in c F_{\text {loc }}^{0}(U)$ (Dirichlet boundary condition along $\partial U$ ),

(i) $h>0$ on $U$.

(ii) $\mathrm{A}(U, W)$-harmonic profile for $\mathcal{E}-\lambda$ is a function $h$ on $W$ such that

(a) $h$ is $\mathcal{E}-\lambda$-harmonic in $W$,

(b) $h \in \mathcal{F}_{\text {loc }}^{0}(U, W)$ (Dirichlet boundary condition along $\partial U$ ),

(c) $h>0$ on $W$.

For any harmonic profile $h$, we set $h(x):=0$ for all $x \in X \backslash U$. Harmonic profiles can be constructed using Green functions and the boundary Harnack principle. In [20, Theorem 4.16] the construction of an $\mathcal{E}$-harmonic profile is given for unbounded inner uniform domains in a Harnack-type Dirichlet space. In fact, in this case the $\mathcal{E}$-harmonic profile is unique up to multiplication by a scalar. The existence and uniqueness of an $\mathcal{E}$-harmonic profile for unbounded inner uniform domains in a metric measure Dirichlet space with length metric and satisfying $\mathrm{VD}, \operatorname{PI}(\Psi), \mathrm{CSA}(\Psi)$ will be given in [22].

Bounded inner uniform domains do not have an $\mathcal{E}$-harmonic profile due to the maximum principle. Instead one may use the ground state which is a an $\mathcal{E}-\lambda$-harmonic profile when $\lambda$ is the principle Dirichlet eigenvalue. 
We will use $(U, W)$-harmonic profiles when the domain is bounded inner uniform or only locally inner uniform (cf. Definition 1.10). For instance a Green function on $U$ with pole in $U \backslash \bar{W}$ would be a $(U, W)$-harmonic profile; see also [27, Proposition 5.9].

The next lemma is a special case of [27, Lemma 6.6], we include the proof for the convenience of the reader.

Lemma 2.6 Let $\lambda \in \mathbb{R}$ and let $h$ be a $(U, W)$-profile for $\mathcal{E}-\lambda(\cdot, \cdot)$. Then

$$
\forall f \in \mathcal{F}^{h}, \quad\|h f\|_{\mathcal{F}}^{2}-\lambda \int f^{2} h^{2} d \mu=\int h^{2} d \Gamma(f, f)+\int f^{2} h^{2} d \mu=\|f\|_{\mathcal{F} h}^{2} .
$$

In particular, $H^{-1}\left(\mathcal{F}^{0}(W)\right)=\mathcal{F}^{h}$.

Proof We first show that Eq. 8 holds. Indeed, this easily follows from the $\mathcal{E}-\lambda$-harmonicity of $h$ provided that $f \in \mathcal{F}_{\mathrm{c}}(W) \cap L^{\infty}$.

For general $f \in \mathcal{F}^{h}$, we can find a sequence $\left(f_{n}\right) \subset \mathcal{F}_{\mathrm{c}}(W) \cap L^{\infty}$ such that $f_{n} \rightarrow f$ in $\left(\mathcal{F}^{h},\|\cdot\|_{\mathcal{F}^{h}}\right)$, by Lemma 2.2. Applying again the $\mathcal{E}$-harmonicity of $h$, we see that $\left(h f_{n}\right)$ is a Cauchy sequence in $\left(\mathcal{F}^{0}(W),\|\cdot\|_{\mathcal{F}}\right)$ with $L^{2}$-limit $h f$. The closedness of $\left(\mathcal{E}_{W}^{D}, \mathcal{F}^{0}(W)\right)$ now yields that $h f \in \mathcal{F}^{0}(W)$ and $h f_{n} \rightarrow h f$ in $\left(\mathcal{F}^{0}(W),\|\cdot\|_{\mathcal{F}}\right)$. Together with Lemma 2.2, we see that the two spaces $H^{-1}\left(\mathcal{F}^{0}(W)\right)$ and $\mathcal{F}^{h}$ coincide.

\subsection{Doob's $h$-transform on Locally Inner Uniform Domains}

Assume that

(i) $U$ is locally $\left(c_{u}, C_{u}\right)$-inner uniform up to scale $R_{0}$ near $W$ (in the sense of Definition $1.10)$,

(ii) A2 and CSA $(\Psi)$ hold on $X$,

(iil) VD, RVD, and $\mathrm{PI}(\Psi)$ hold locally on $\left\{x \in X: d(x, w) \leq R_{0}\right.$ for some $\left.w \in W\right\}$ up to scale $R_{0}$.

Proposition 2.7 Let $h$ be a $(U, W)$-profile for $\mathcal{E}$. Then there are constants $a_{0}, A_{0} \in(0, \infty)$ such that the Dirichlet space $\left(W^{\sharp}, d_{U}, h^{2} d \mu, \mathcal{E}^{h}, \mathcal{F}^{h}\right)$ satisfies $V D$ and $P I(\Psi)$ locally on any ball $B_{\widetilde{U}}\left(x, a_{0} R\right)$ up to scale $a_{0} R$, provided that $B_{\widetilde{U}}\left(x, A_{0} R\right) \subset W^{\sharp}$ and $a_{0} R \in\left(0, R_{0}\right)$.

Proof The volume doubling property of the measure $h^{2} d \mu$ follows from the doubling property of $\mu$ on $(X, d)$, the elliptic Harnack inequality EHI and, for inner balls near the boundary of $U$, the Carleson estimate of Corollary 1.14, both applied to $h$, by the same reasoning as in [20, Proof of Theorem 4.17] or [27, Theorem 6.12].

The Poincaré inequality follows by the same reasoning as in [20, Theorem 3.13 , Theorem 3.27] or [27, Theorem 6.12]. Here, we only give a sketch of the proof that may guide the reader through the above references.

For inner balls $B_{\widetilde{U}}\left(x, A_{0} R\right) \subset W$ (away from the boundary of $U$ ), it is easily seen that the Poincaré inequality is satisfied by $\left(\mathcal{E}^{h}, \mathcal{F}^{h}\right)$ on $B_{U}\left(x, a_{0} R\right)$. Indeed, it suffices to replace the measure $\mu$ by $h^{2} d \mu$ in the Poincaré inequality satisfied by $(\mathcal{E}, \mathcal{F}$ ) (adjusting the Poincaré constant if necessary). This is possible because the elliptic Harnack inequality EHI applies to the harmonic profile $h$.

To prove that $\left(\mathcal{E}^{h}, \mathcal{F}^{h}\right)$ satisfies the Poincaré inequality on inner balls $B=B_{\widetilde{U}}\left(x, a_{0} R\right)$ that are close to the boundary of $U$, we choose a Whitney cover for $B$. A Whitney cover for $B$ is a collection of balls $B_{i}=B\left(x_{i}, r_{i}\right)$ such that (1) the balls $B\left(x_{i}, 2 r_{i}\right)$ cover $B$, (2) $r_{i}=\epsilon d_{U}\left(B_{i}, \partial U\right)$ for all $i$ for some fixed small $\epsilon>0$, and (3) each point $x \in B$ is 
contained in at most $N$ of the balls $B\left(x_{i}, 8 r_{i}\right)$, for some integer $N$ independent of $x$. The construction of a Whitney cover (see, e.g., [31, Section 5.3]) uses the local compactness and the volume doubling property of the metric measure space $\left(W^{\sharp}, d_{U}, h^{2} d \mu\right)$. It can then be verified that the Poincare inequalities on each of the balls $\left(B_{i}\right)$ can be combined to obtain the Poincare inequality on $B$. This step makes use of the volume doubling property and the fact that $d_{U}$ is a length metric.

A proof of the following proposition in the special case when $U=W$ is an unbounded inner uniform domain and $R_{0}=\infty$ was obtained in collaboration of the author with N. Kajino, and will be given in [22].

Proposition 2.8 Let $h$ be a $(U, W)$-profile for $\mathcal{E}$. Then there are constants $a_{0}, A_{0} \in(0, \infty)$ such that the Dirichlet space $\left(W^{\sharp}, d_{U}, h^{2} d \mu, \mathcal{E}^{h}, \mathcal{F}^{h}\right)$ satisfies $C S A(\Psi)$ locally on any ball $B_{\widetilde{U}}\left(x, a_{0} R\right)$ up to scale $a_{0} R$, provided that $B_{\widetilde{U}}\left(x, A_{0} R\right) \subset W^{\sharp}$ and $a_{0} R \in\left(0, R_{0}\right)$.

Proof Let $a_{0}=\frac{1}{12 C_{U}}$ and $A_{0}=3 C_{U}$, where $C_{U}$ is as in Lemma 1.15. Consider an inner ball $B_{\widetilde{U}}\left(x, 3 C_{U} R\right) \subset W^{\sharp}$, where $R \in\left(0, R_{0} /\left(12 C_{U}\right)\right)$. The inner ball $B_{\widetilde{U}}(x, R)$ can be embedded into the ball $B(p(x), R)$ in $(X, d)$ by the canonical projection $p: \widetilde{U} \rightarrow \bar{U}$. On the underlying space $(X, d, \mu, \mathcal{E}, \mathcal{F}), \operatorname{CSA}(\Psi)$ is satisfied by assumption. Thus, for $c_{1}>0$ (to be chosen later) there exists a cutoff function $\phi$ for $B(p(x), R)$ with compact support $D:=\operatorname{supp}(\phi)$ in $B(p(x), 2 R)$ such that

$$
\forall f \in \mathcal{F}, \quad \int_{A} f^{2} d \Gamma(\phi, \phi) \leq c_{1} \int_{A} \phi^{2} d \Gamma(f, f)+\frac{c_{2}}{\Psi(R)} \int_{A} f^{2} d \mu,
$$

where $A=B(p(x), 2 R) \backslash B(p(x), R)$. Let $D^{\prime}$ be the connected component of $p^{-1}(D \cap \bar{U})$ in $\left(\widetilde{U}, d_{U}\right)$ that contains $x$. By Lemma $1.15, D^{\prime} \subset B_{\widetilde{U}}\left(x, 2 C_{U} R\right)=B_{\widetilde{U}}(x, R+r)$ for $r=$ $2 C_{U} R-R$. Note that $D^{\prime}$ is compact and the $d_{U}$-distance between $D^{\prime}$ and the other connected components $p^{-1}(D \cap \bar{U}) \backslash D^{\prime}$ is greater than $2 \eta$ for any sufficiently small $\eta \in(0,1)$. Due to the regularity proved in Proposition 2.4 , there exists a function $g_{D^{\prime}} \in \mathcal{C}_{\mathrm{c}}\left(W^{\sharp}\right) \cap \mathcal{F}^{h}$ taking values in $[0,1]$ such that $g_{D^{\prime}}=1$ on the open $\eta$-neighborhood of $D^{\prime}$ in $\left(W^{\sharp}, d_{U}\right)$ and $g_{D^{\prime}}=0$ outside the $2 \eta$-neighborhood of $D^{\prime}$ in $\left(W^{\sharp}, d_{U}\right)$, in particular $g_{D^{\prime}}=0$ on $p^{-1}(D \cap \bar{U}) \backslash D^{\prime}$. We have $h g_{D^{\prime}} \in \mathcal{F}^{0}(W)$ by Lemma 2.6. By Lemma 2.3, $\left.h g_{D^{\prime}} \phi\right|_{W}$ is in $\mathcal{F}^{0}(W)$. In particular, the function

$$
\psi(z):=\phi(z) g_{D^{\prime}}(z)=\phi(z) 1_{D^{\prime}}(z), \quad z \in W,
$$

is in $\mathcal{F}^{h}$. It is clear that $\psi$ takes values in $[0,1]$, that $\psi=1$ on $B_{U}(x, R)$, and that $\psi$ has compact support in $B_{\widetilde{U}}(x, R+r)$. Moreover, $\psi$ is continuous in $W$ and extends continuously to the boundary $W^{\sharp} \backslash W$.

It suffices to show that there exist constants $c_{3}, c_{4}>0$ such that

$$
\begin{aligned}
& \int_{A_{U}} f^{2} h^{2} d \Gamma(\psi, \psi) \\
& \quad \leq c_{3} \int_{B_{U}(x, R+r) \backslash B_{U}\left(x, R-\frac{1}{100} r\right)} \psi^{2} h^{2} d \Gamma(f, f)+\frac{c_{4}}{\Psi(r)} \int_{B_{U}(x, R+r) \backslash B_{U}\left(x, R-\frac{1}{100} r\right)} f^{2} h^{2} d \mu,
\end{aligned}
$$

for all $f \in \mathcal{F}^{h}$, where $A_{U}=B_{U}(x, R+r) \backslash B_{U}(x, R)$. It suffices to show (10) for bounded $f \in \mathcal{F}^{h}$. Indeed, we can then apply (10) to $f_{n}:=(f \wedge n) \vee(-n)$ and, letting $n \rightarrow \infty$ and using Fatou's lemma, obtain (10) for general $f \in \mathcal{F}^{h}$.

Note that $A_{U} \subset A \cap U$. Due to the Dirichlet boundary condition of $h$, the energy measure $h^{2} d \Gamma$ does not charge $X \backslash U$. The positivity of $h^{2} d \Gamma(\psi, \psi)$ and strong locality then yield

$$
\int_{A_{U}} f^{2} h^{2} d \Gamma(\psi, \psi) \leq \int_{A \cap U \cap D^{\prime}} f^{2} h^{2} d \Gamma\left(\phi 1_{D^{\prime}}, \phi 1_{D^{\prime}}\right) \leq \int_{A} f^{2} g_{D^{\prime}}^{2} h^{2} d \Gamma(\phi, \phi) .
$$


We will apply (9) to the right side of the above estimate. We also use the Leibniz rule for the energy measure, strong locality and the fact that $g_{D^{\prime}}$ is constant on each connected component of the support of $\phi$, and the Cauchy-Schwarz inequality. We obtain

$$
\begin{aligned}
& \int_{A_{U}} f^{2} h^{2} d \Gamma(\psi, \psi) \\
& \leq c_{1} \int_{A} \phi^{2} d \Gamma\left(f g_{D^{\prime}} h, f g_{D^{\prime}} h\right)+\frac{c_{2}}{\Psi(R)} \int_{A} f^{2} g_{D^{\prime}}^{2} h^{2} d \mu \\
& \leq c_{1} \int_{A} \phi^{2} g_{D^{\prime}}^{2} d \Gamma(f h, f h)+\frac{c_{2}}{\Psi(R)} \int_{A} f^{2} h^{2} d \mu \\
& \leq 2 c_{1} \int_{A_{\eta}} \phi^{2} g_{D^{\prime}}^{2} h^{2} d \Gamma(f, f)+2 c_{1} \int_{A_{\eta}} \phi^{2} g_{D^{\prime}}^{2} f^{2} d \Gamma(h, h)+\frac{c_{2}}{\Psi(R)} \int_{A_{\eta}} f^{2} h^{2} d \mu,
\end{aligned}
$$

where $A_{\eta}:=B_{U}(x, R+r+2 \eta) \backslash B_{U}(x, R)$. We let $\eta \rightarrow 0$ and get the above estimate with $A_{U}$ in place of $A_{\eta}$,

$$
\begin{aligned}
& \int_{A_{U}} f^{2} h^{2} d \Gamma(\psi, \psi) \\
& \quad \leq 2 c_{1} \int_{A_{U}} \psi^{2} h^{2} d \Gamma(f, f)+2 c_{1} \int_{A_{U}} \psi^{2} f^{2} d \Gamma(h, h)+\frac{c_{2}}{\Psi(R)} \int_{A_{U}} f^{2} h^{2} d \mu,
\end{aligned}
$$

At this point we can conclude that the cutoff Sobolev inequality holds on balls for the inner metric (rather than on annuli). Indeed, by the product rule and Cauchy-Schwarz inequality for the energy measure and the $\mathcal{E}$-harmonicity of $h$, we have

$$
\begin{aligned}
\int_{A_{U}} \psi^{2} f^{2} d \Gamma(h, h) & \leq \int \psi^{2} f^{2} d \Gamma(h, h)=\int d \Gamma\left(h, h \psi^{2} f^{2}\right)-2 \int_{A_{U}} \psi f d \Gamma(h, h \psi f) \\
& \leq \frac{1}{2} \int \psi^{2} f^{2} d \Gamma(h, h)+4 \int \psi^{2} h^{2} d \Gamma(f, f)+4 \int f^{2} h^{2} d \Gamma(\psi, \psi),
\end{aligned}
$$

hence the cutoff Sobolev inequality on balls for the inner metric follows by putting the above estimate into Eq. 11 and rearranging terms.

Let $\delta=\frac{r}{100}$. For $x_{i} \in A_{U}$, let $\phi_{i}$ be a cutoff function for $B_{U}\left(x_{i}, \tau \delta\right)$ in $B_{U}\left(x_{i}, \delta\right)$ which satisfies the cutoff Sobolev inequality on balls for a given $\epsilon=\frac{1}{32}$. The parameter $\tau \in(0,1)$ is provided by the cutoff Sobolev inequality on balls for the inner metric, which we proved above. We choose the points $x_{i}$ in such a way $A_{U} \subset \bigcup_{i} B_{U}\left(x_{i}, \tau \delta\right)$ and no more than $N$ of the balls $B_{U}\left(x_{i}, \delta\right)$ intersect in any given point, where $N$ depends only on the constants in VD and RVD. Observe that $\sum_{i} \phi_{i}^{2} \geq 1$ on $A_{U}$.

By the $\mathcal{E}$-harmonicity of $h$, the Cauchy-Schwarz inequality and the product rule for the energy measure,

$$
\begin{aligned}
\int_{A_{U}} \psi^{2} f^{2} d \Gamma(h, h) & \leq \sum_{i} \int \phi_{i}^{2} \psi^{2} f^{2} d \Gamma(h, h) \\
& =\sum_{i} \int d \Gamma\left(h, h \phi_{i}^{2} \psi^{2} f^{2}\right)-2 \sum_{i} \int \phi_{i} \psi f h d \Gamma\left(h, \phi_{i} \psi f\right) \\
& \leq \frac{1}{2} \sum_{i} \int \phi_{i}^{2} \psi^{2} f^{2} d \Gamma(h, h)+4 \sum_{i} \int \psi^{2} f^{2} h^{2} d \Gamma\left(\phi_{i}, \phi_{i}\right) \\
& \quad+8 \sum_{i} \int \phi_{i}^{2} f^{2} h^{2} d \Gamma(\psi, \psi)+8 \sum_{i} \int \phi_{i}^{2} \psi^{2} h^{2} d \Gamma(f, f) .
\end{aligned}
$$


Rearranging, using the fact that $\sum_{i} \phi_{i}^{2} \leq N$, and applying the cutoff Sobolev inequality for each of the cutoff functions $\phi_{i}$, as well as the Cauchy-Schwarz inequality and the product rule for the energy measure,

$$
\begin{aligned}
& \quad \sum_{i} \int \phi_{i}^{2} \psi^{2} f^{2} d \Gamma(h, h) \\
& \leq 16 N \int_{B_{U}(x, R+r) \backslash B_{U}(x, R-\delta)} f^{2} h^{2} d \Gamma(\psi, \psi)+16 N \int_{B_{U}(x, R+r) \backslash B_{U}(x, R-\delta)} \psi^{2} h^{2} d \Gamma(f, f) \\
& \quad+16 \epsilon \sum_{i} \int \phi_{i}^{2} \psi^{2} f^{2} d \Gamma(h, h)+32 N \epsilon \int_{B_{U}(x, R+r) \backslash B_{U}(x, R-\delta)} f^{2} h^{2} d \Gamma(\psi, \psi) \\
& \quad+32 N \epsilon \int_{B_{U}(x, R+r) \backslash B_{U}(x, R-\delta)} \psi^{2} h^{2} d \Gamma(f, f) \\
& \quad+8 N \frac{C_{0} \epsilon^{1-\beta_{2} / 2}}{\Psi((1-\tau) \delta)} \int_{B_{U}(x, R+r) \backslash B_{U}(x, R-\delta)} \psi^{2} f^{2} h^{2} d \mu .
\end{aligned}
$$

Because $\psi=1$ on $B_{U}(x, R)$, the energy measure $d \Gamma(\psi, \psi)$ does not charge $B_{U}(x, R)$ by strong locality. Choosing $\epsilon=\frac{1}{32}$ and rearranging once again,

$$
\begin{aligned}
& \int_{A_{U}} \psi^{2} f^{2} d \Gamma(h, h) \\
\leq & 34 N \int_{A_{U}} f^{2} h^{2} d \Gamma(\psi, \psi)+34 N \int_{B_{U}(x, R+r) \backslash B_{U}(x, R-\delta)} \psi^{2} h^{2} d \Gamma(f, f) \\
& +16 N \frac{C_{0} 2^{-5+5 \beta_{2} / 2}}{\Psi((1-\tau) \delta)} \int_{B_{U}(x, R+r) \backslash B_{U}(x, R-\delta)} \psi^{2} f^{2} h^{2} d \mu .
\end{aligned}
$$

Combining this with Eq. 11,

$$
\begin{aligned}
& \left(1-2 c_{1} 34 N\right) \int_{A_{U}} f^{2} h^{2} d \Gamma(\psi, \psi) \\
\leq & 2 c_{1}(1+34 N) \int_{B_{U}(x, R+r) \backslash B_{U}(x, R-\delta)} \psi^{2} h^{2} d \Gamma(f, f) \\
+ & \frac{c_{2}}{\Psi(R)} \int_{A_{U}} f^{2} h^{2} d \mu+2 c_{1} 16 N \frac{C_{0} 2^{-5+5 \beta_{2} / 2}}{\Psi((1-\tau) \delta)} \int_{B_{U}(x, R+r) \backslash B_{U}(x, R-\delta)} \psi^{2} f^{2} h^{2} d \mu .
\end{aligned}
$$

Note that, by Eq. 3, $\frac{c_{2}}{\Psi(R)} \leq C_{\Psi}\left(2 C_{U}-1\right)^{\beta_{2}} \frac{c_{2}}{\Psi(r)}$, and $\frac{1}{\psi((1-\tau) \delta)} \leq C_{\Psi}\left(\frac{100}{1-\tau}\right)^{\beta_{2}} \frac{1}{\Psi(r)}$. Choosing $c_{1}>0$ sufficiently small, we obtain the desired estimate (10).

Proposition 2.9 Let $h$ be a $(U, W)$-profile for $\mathcal{E}$. Then there are constants $a_{0}, A_{0} \in(0, \infty)$ such that the Dirichlet space $\left(W^{\sharp}, d_{U}, h^{2} d \mu, \mathcal{E}^{h}, \mathcal{F}^{h}\right)$ satisfies $P H I(\Psi)$ locally on any ball $B_{\widetilde{U}}\left(x, a_{0} R\right)$ up to scale $a_{0} R$, provided that $B_{\widetilde{U}}\left(x, A_{0} R\right) \subset W^{\sharp}$ and $a_{0} R \in\left(0, R_{0}\right)$. Moreover, for any $\lambda \in \mathbb{R}, P H I(\Psi)$ holds on these balls for $\left(\mathcal{E}^{h}-\lambda(\cdot h, \cdot h)\right)$-harmonic functions in place of $\mathcal{E}^{h}$-harmonic functions. The constant in the parabolic Harnack inequality then also depends on an upper bound for $|\lambda| \Psi\left(R_{0}\right)$.

Proof This proposition follows from applying [24, Theorem 5.3] (with constant $C_{3}=|\lambda|$ ) together with Propositions 2.4, 2.7, 2.8. 
Proposition 2.10 Let $\lambda \in \mathbb{R}$ and let $\hat{h}$ be a $(U, W)$-profile for $(\mathcal{E}-\lambda(\cdot, \cdot))$. Then there are constants $a_{0}, A_{0} \in(0, \infty)$ such that the Dirichlet space $\left(W^{\sharp}, d_{U}, \hat{h}^{2} d \mu, \mathcal{E}^{\hat{h}}, \mathcal{F}^{\hat{h}}\right)$ satisfies $V D, P I(\Psi), C S A(\Psi), P H I(\Psi)$ locally on any ball $B_{\widetilde{U}}\left(x, a_{0} R\right)$ up to scale $a_{0} R$, provided that $B_{\widetilde{U}}\left(x, A_{0} R\right) \subset W^{\sharp}$ and $a_{0} R \in\left(0, R_{0}\right)$. The constants in these conditions depend on an upper bound for $|\lambda| \Psi\left(R_{0}\right)$.

Moreover, if $h$ is a $(U, W)$-profile for $\mathcal{E}$, then

$$
C^{-1} \frac{\hat{h}(z)}{h(z)} \leq \frac{\hat{h}(y)}{h(y)} \leq C \frac{\hat{h}(z)}{h(z)}, \quad \text { for all } y, z \in B_{\widetilde{U}}\left(x, a_{0} R\right) .
$$

with a constant $C \in(0, \infty)$ depending on an upper bound for $|\lambda| \Psi\left(R_{0}\right)$.

Proof Let $h$ be a $(U, W)$-profile for $\mathcal{E}$. By Proposition 2.9, $\left(\mathcal{E}^{h}+\lambda(\cdot h, \cdot h)\right)$ satisfies the parabolic Harnack inequality, hence also the elliptic Harnack inequality. Since $\hat{h} / h$ is $\left(\mathcal{E}^{h}+\right.$ $\lambda(\cdot h, h)$ )-harmonic on $W^{\sharp}$, the EHI yields (12). Together with Proposition 2.4, Proposition 2.7 and Proposition 2.8, we obtain that $\left(W^{\sharp}, d_{U}, \hat{h}^{2} d \mu, \mathcal{E}^{\hat{h}}, \mathcal{F}^{\hat{h}}\right)$ is a regular Dirichlet space and satisfies $\mathrm{VD}, \operatorname{PI}(\Psi), \mathrm{CSA}(\Psi)$ locally on $B_{\widetilde{U}}\left(x, a_{0} R\right)$ up to scale $a_{0} R$. The parabolic Harnack inequality follows by applying [24, Theorem 5.3].

\section{Main Results: Estimates for Symmetric Dirichlet Heat Kernels}

Let $(X, d, \mu, \mathcal{E}, \mathcal{F})$ be a (symmetric, strongly local, regular) metric measure Dirichlet space as in Section 1.1. We fix a domain $U \subset X$ and a subdomain $W \subset U$.

Let

$$
\Phi(R, t):=\sup _{s>0}\left\{\frac{R}{s}-\frac{t}{\Psi(s)}\right\} .
$$

Theorem 3.1 Let $x, y \in U$ and $T>0$. Assume that

(i) $U$ is locally $\left(c_{u}, C_{u}\right)$-inner uniform near $B_{x}:=B_{U}\left(x, R_{x}\right)$ up to scale $R_{x}$ and near $B_{y}:=B_{U}\left(y, R_{y}\right)$ up to scale $R_{y}$.

(ii) $C S A(\Psi)$ holds on $X$, and $(X, d)$ is geodesic.

(iii) $A 2-Y, V D, R V D$, and $P I(\Psi)$ hold locally on $B\left(x, R_{x}\right)$ up to scale $R_{x}$ and on $B\left(y, R_{y}\right)$ up to scale $R_{y}$.

(iv) There exist $\lambda \in \mathbb{R}$ and a function $h$ that is positive continuous in $U$ and both $a$ $\left(U, B_{x}\right)$-profile and $a\left(U, B_{y}\right)$-profile for $(\mathcal{E}+\lambda(\cdot, \cdot))$.

Then there are constants $a_{0} \in(0,1)$ and $A_{1}, a_{2}, a_{3}, A_{4} \in(0, \infty)$ such that the Dirichlet heat kernel $p_{U}^{D}$ exists

and satisfies the upper bound

$$
p_{U}^{D}(t, \xi, \zeta) \leq \frac{A_{1} h(\xi) h(\zeta)}{V\left(\xi, \tau_{x}\right)^{\frac{1}{2}} V\left(y, \tau_{y}\right)^{\frac{1}{2}} h\left(\xi^{\prime}\right) h\left(\zeta^{\prime}\right)} \exp \left(-\frac{1}{2} \Phi\left(d_{U}(x, y), a_{2} t\right)\right),
$$

for all $t \in(0, T), \xi \in B_{\widetilde{U}}\left(x, a_{0} R_{x}\right), \zeta \in B_{\widetilde{U}}\left(y, a_{0} R_{y}\right)$, and the near-diagonal lower bound

$$
p_{U}^{D}(t, \xi, \zeta) \geq \frac{a_{3} h(\xi) h(\zeta)}{V\left(\xi, \tau_{x}\right)^{\frac{1}{2}} V\left(y, \tau_{y}\right)^{\frac{1}{2}} h\left(\xi^{\prime}\right) h\left(\zeta^{\prime}\right)} \exp \left(-\frac{1}{2} \Phi\left(d_{U}(x, y), A_{4} t\right)\right) .
$$

for all $t \in(0, T), \xi \in B_{\widetilde{U}}\left(x, a_{0} R_{x}\right), \zeta \in B_{\widetilde{U}}\left(y, a_{0} R_{y}\right)$ whenever $d(x, y) \leq R_{x} / 4$. Here, $\tau_{x}=\Psi^{-1}(t) \wedge a_{0} R_{x}, \tau_{y}=\Psi^{-1}(t) \wedge a_{0} R_{y}$, and $\xi^{\prime}, \zeta^{\prime}$ are points in $U$ such that $d_{U}\left(\xi, \xi^{\prime}\right)=$ $\tau_{x} / 4, d_{U}\left(\xi^{\prime}, \partial U\right) \geq c_{u} \tau_{x} / 8$, and $d_{U}\left(\zeta, \zeta^{\prime}\right)=\tau_{y} / 4, d_{U}\left(\zeta^{\prime}, \partial U\right) \geq c_{u} \tau_{y} / 8$. 
The constant $a_{0}$ depends only on $\left(c_{u}, C_{u}\right)$. The constants $A_{1}, a_{2}, a_{3}, A_{4}$ depend only on $C_{\Psi}, \beta_{1}, \beta_{2}$, the constants in $V D, R V D, P I(\Psi), C S A(\Psi)$, upper bounds for $|\lambda|\left(1 \vee \Psi\left(R_{x}\right) \vee\right.$ $\left.\Psi\left(R_{y}\right)\right), T / \Psi\left(R_{x}\right)$ and $T / \Psi\left(R_{y}\right)$.

Proof The upper bound follows from [24, Theorem 6.6] applied to the $h$-transformed kernel $p_{h}$. The near-diagonal lower bound follows from [24, Corollary 6.10].

Remark 3.2 A full lower bound (for any $x, y \in X$ ) can be obtained provided that (i) - (iv) hold for all $x \in X$ up to a fixed scale $R_{x}=R_{0}$. This follows from the near-diagonal lower bound and from applying $\mathrm{PHI}(\Psi)$ repeatedly along a Harnack chain of balls connecting $x$ to $y$. This argument uses the fact that $d_{U}$ is a length metric.

In the special case when $U$ is unbounded and $R_{0}=\infty$, the global hypotheses A2, VD, RVD, PI $(\Psi), \mathrm{CSA}(\Psi)$ can equivalently be replaced by A2, VD, RVD and two-sided estimates for the global heat kernel on $X$ thanks to [18, Theorem 1.2]. The principle Dirichlet eigenvalue $\lambda$ equals zero in this case. The global Dirichlet heat kernel estimates in this special case will be the main result of the forthcoming paper [22] by N. Kajino.

For bounded domains, we have the following result.

\section{Theorem 3.3 Assume that}

(i) $U$ is bounded and locally $\left(c_{u}, C_{u}\right)$-inner uniform up to scale $R_{0}$.

(ii) $C S A(\Psi)$ holds on $X$, and $(X, d)$ is geodesic.

(iii) $A 2-Y, V D, R V D$, and $P I(\Psi)$ hold locally on $Y=\left\{x \in X: d(x, U) \leq R_{0}\right\}$ up to scale $R_{0}$.

Let $\lambda>0$ be the principal Dirichlet eigenvalue for $-L_{U}^{D}$ and $\phi$ the associated positive $L^{2}$ normalized eigenfunction. Then the Dirichlet heat kernel $p_{U}^{D}$ on $U$ satisfies the two-sided bounds

$$
\begin{aligned}
& p_{U}^{D}(t, x, y) \geq a_{1} \frac{\phi(x) \phi(y)}{\int_{B_{U}\left(x, \Psi^{-1}(t)\right)} \phi^{2} d \mu} \exp \left(-\frac{1}{2} \Phi\left(d_{U}(x, y), A_{1} t\right)\right), \\
& p_{U}^{D}(t, x, y) \leq A_{2} \frac{\phi(x) \phi(y)}{\int_{B_{U}\left(x, \Psi^{-1}(t)\right)} \phi^{2} d \mu} \exp \left(-\frac{1}{2} \Phi\left(d_{U}(x, y), a_{2} t\right)\right),
\end{aligned}
$$

for all $t \in\left(0, \Psi\left(R_{0}\right)\right), x, y \in \widetilde{U}$, and

$$
a_{3} \leq \frac{p_{U}^{D}(t, x, y) e^{t \lambda}}{\phi(x) \phi(y)} \leq A_{3}, \quad \forall t \geq \Psi\left(R_{0}\right), \forall x, y \in \widetilde{U} .
$$

The constants $A_{1}, a_{1}, A_{2}, a_{2}, A_{3}, a_{3} \in(0, \infty)$ depend only on $c_{u}, C_{u}, C_{\Psi}, \beta_{1}, \beta_{2}$, on an upper bound for diam $(U) / R_{0}$, and on the constants in VD, RVD, PI( $\left.\Psi\right), C S A(\Psi)$.

Proof We proved in Proposition 2.10 that $\left(\tilde{U}, d_{U}, \phi^{2} d \mu, \mathcal{E}^{\phi}, \mathcal{F}^{\phi}\right)$ is a symmetric strongly local regular metric measure Dirichlet space which satisfies $\operatorname{VD}, \operatorname{PI}(\Psi), \operatorname{CSA}(\Psi)$ and $\operatorname{PHI}(\Psi)$. Clearly, $\left(\widetilde{U}, d_{U}\right)$ is geodesic. Applying [24, Theorem 6.6], we obtain sub-Gaussian upper bounds for the $\phi$-transformed kernel

$$
p_{\phi}(t, x, y)=\frac{p_{U}^{D}(t, x, y) e^{t \lambda}}{\phi(x) \phi(y)}, \quad x, y \in \tilde{U}, t>0 .
$$

The lower bounds follow from [24, Corollary 6.10] (when $d(x, y)$ is small) together with $\operatorname{PHI}(\Psi)$ (when $d(x, y)$ is large). Though the constants initially depend also on $\lambda \Psi\left(R_{0}\right)$, this 
quantity can be estimated in terms of the other constants because here $\lambda$ is the eigenvalue. Indeed, it suffices to apply the Courant-Fisher min-max principle and use the cutoff function given by $\operatorname{CSA}(\Psi)$ as test function.

We have the following two corollaries. We omit the proofs since they are almost identical to the proofs of [27, Corollary 7.10] and [27, Proof of Corollary 7.12].

Corollary 3.4 Under the hypotheses and notation of Theorem 3.3, there exists a bounded continuous function $w$ on $U$ and a real number $\omega>0$ such that

$$
\forall t \geq \Psi\left(R_{0}\right), x, y \in U, \quad\left|\frac{p_{U}^{D}(t, x, y) e^{\lambda t}}{\phi(x) \phi(y) w(y)}-1\right| \leq A_{4} e^{-\omega t},
$$

for some constant $A_{4} \in(0, \infty)$. Further, $a_{3} \leq w \leq A_{3}$, and

$$
A_{4} \leq \frac{A_{3}}{a_{3}\left(1-a_{3} / A_{3}\right)^{2}}, \quad \text { and } \quad \omega \geq \frac{1}{\Psi\left(R_{0}\right)} \log \left(\frac{1}{1-a_{3} / A_{3}}\right),
$$

where $a_{3}, A_{3}, R$ are as in Theorem 3.3.

Corollary 3.5 Under the hypotheses and notation of Theorem 3.3, there exists a constant $A_{5} \in(0, \infty)$ such that, if $\psi \neq \phi$ is an $L^{2}$-normalized eigenfunction of $-L_{U}^{D}$ with eigenvalue $\lambda_{\psi}$, then $\eta:=\lambda_{\psi}-\lambda \geq 1 /\left(A_{5} \Psi\left(R_{0}\right)\right)$ and

$$
\forall x \in U, \quad|\psi(x)| \leq \frac{A_{5} \phi(x)}{V\left(x, \eta^{-1 / 2}\right)^{1 / 2} \phi\left(x^{\prime}\right)},
$$

where $x^{\prime}$ is a point in $U$ such that $d_{U}\left(x, x^{\prime}\right)=\eta^{-1 / 2} / 4$ and $d_{U}\left(x^{\prime}, \partial U\right) \geq c_{u} \eta^{-1 / 2} / 8$.

\section{Examples}

\subsection{Sierpinski Gasket}

Consider the standard Sierpinski gasket $S$. For the construction of the symmetric strongly local regular Dirichlet form associated with the Laplacian on the standard Sierpinski gasket $S$, see e.g., [3]. Let $F_{n}$ be the finite set of vertices in the graph approximation $S_{n}$ of the Sierpinski gasket at level $n$. We set $F_{0}=\left\{s_{1}, s_{2}, s_{3}\right\}$.

Proposition 4.1 Let L be a line in $\mathbb{R}^{2}$ that intersects the Sierpinski gasket and let $U^{+}$and $U^{-}$be the domains in the Sierpinski gasket that lie to either side of the line. Let $U$ be either $U^{+}$or $U^{-}$. Then $U$ is an inner uniform domain in the Sierpinski gasket if and only if

(C) there exists a constant $c_{0} \in(0,1)$ such that for every triangular hole of side length $l$ of $U$, the distance between the line and the hole is either 0 or greater than $c_{0} l$.

The proof relies on constructing John paths which are defined as follows. We say that a curve $\gamma:[0, l] \rightarrow U$ is a $c$-John path in $U$ if for any $t \in[0, l]$,

$$
d(\gamma(t), \partial U) \geq c t .
$$

Proof Without loss of generality we assume that $S$ is scaled to have side length 1 . 
Suppose condition (C) is satisfied. Pick two arbitrary points $x, y \in U$. Let $m$ be the largest integer such that $x$ and $y$ are contained in the same cell of side length $2^{-m}$. Then $x$ and $y$ lie in different cells of side length $2^{-m-1}$. Therefore, any path from $x$ to $y$ in $\left(U, d_{U}\right)$ must pass through at least one vertex $V \in F_{m+1} \cap U$. Since $L$ is a straight line, there exists a geodesic from $x$ to $V$ that lies in $U$, and as we move along this path the distance to the line is an affine function, so the geodesic is a $c$-John path with constant $c$ depending only on $c_{0}$. Similarly, we construct a $c$-John path from $y$ to a vertex $V^{\prime} \in F_{m+1} \cap U$. If $V=V^{\prime}$, then the concatenation of the two John paths yields an inner uniform path from $x$ to $y$. If $V \neq V^{\prime}$, then we include the edge from $V$ to $V^{\prime}$ in our path from $x$ to $y$. This edge is adjacent to a hole whose side length is comparable to $2^{-m-1}$. By condition (C), the line stays away from this edge at scale $2^{-m-1}$. Thus, the path we constructed is indeed inner uniform.

Suppose condition (C) is not satisfied. Then, for any positive integer $n$, we can find two points $x, y \in U$ on different edges of length $2^{-m}$ meeting at a vertex $V$ such that $d_{U}(x, y) / 2^{-m}<\frac{1}{n}$ is small while $d_{U}(x, y) / d(V, L)>n$ is large. Thus, any path connecting $x$ to $y$ in $U$ that satisfies (7) violates the banana condition (6).

Condition (C) is satisfied, for instance, by the domain above the horizontal line at height $k 2^{-n} \frac{\sqrt{3}}{2}$ for any positive integers $k, n$ with $k \leq 2^{n}$, where $\frac{\sqrt{3}}{2}$ is the height of the gasket of side length 1. (C) is also satisfied by the domain above the horizontal line of height $\left(\frac{1}{2}-\sum_{i=1}^{\infty} 2^{-n i}\right) \frac{\sqrt{3}}{2}$ for any integer $n \geq 2$.

It is well known that the semigroup associated with the Laplacian on the Sierpinski gasket admits a heat kernel $p_{t}(x, y)$ which satisfies the two-sided bounds

$$
p_{t}(x, y) \asymp \frac{c_{1}}{\mu\left(B\left(x, t^{-d_{S} / 2}\right)\right)} \exp \left(-\left(\frac{d(x, y)^{d_{w}}}{c_{2} t}\right)^{\frac{1}{d_{w}-1}}\right), \quad x, y \in S, t>0,
$$

where $\mu$ is the Hausdorff measure, $d_{w}=\log 5 / \log 2$ and $d_{s} / 2=\log 3 / \log 5$. See [6]. The heat kernel estimates of Theorem 3.3 apply to the Dirichlet heat kernel on the domains described in Proposition 4.1. It is a consequence of the main result of this paper, Theorem 3.3, that the Dirichlet heat kernel has the same boundary decay rate as any positive harmonic function that vanishes on a portion of the boundary.

The following examples illustrate how to compute boundary decay rates of harmonic functions. These and more general computations are essentially well known. The new contribution of the present paper is that the Dirichlet heat kernel shares the same boundary decay rate as the harmonic functions $h$ studied below. Indeed, the Dirichlet heat kernel estimates of Theorem 3.3 imply that the Dirichlet heat kernel and the ground state $\phi$ share the same boundary decay rate. However, $\phi$ and $h$ share the same boundary decay rate due to a version of the boundary Harnack principle: See [27, Proof of Theorem 6.12] which, thanks to $[24,25]$, generalizes to the present setting of fractal spaces.

The first example is a well-known computation (e.g., [3, Example 3.2.6] or [37, Figure 2.1]) of harmonic functions in the gasket when boundary values are prescribed at the three outer vertices.

Example 4.2 Let $S$ be the Sierpinski gasket. We impose boundary conditions $h\left(s_{1}\right)=a$, $h\left(s_{2}\right)=b, h\left(s_{3}\right)=0$ on the three outer vertices of the gasket. Let $\left.U=S \backslash\left\{s_{1}, s_{2}, s_{3}\right\}\right)$. Then there exists a unique non-negative continuous function $h$ on $S$ that is harmonic on $U$ and satisfies the given boundary condition. Figure 1 shows the values $a_{n}, b_{n}, c_{n}$ that the 


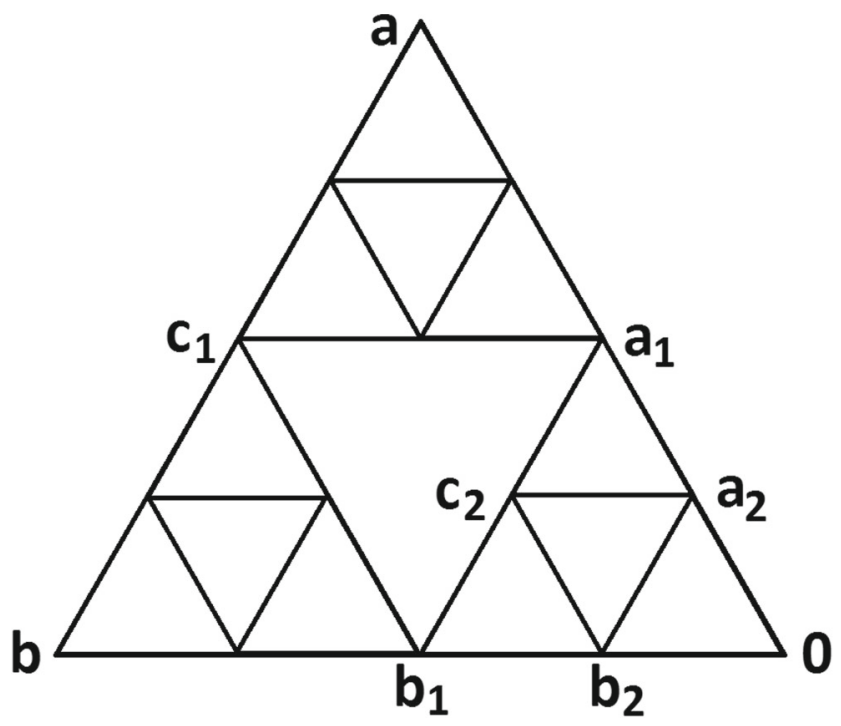

Fig. 1 Harmonic function on Sierpinski gasket with zero boundary condition on one vertex

harmonic function $h$ takes on the vertices in the Sierpinski gasket. These values of $h$ can be computed iteratively using geometric series:

$$
\begin{aligned}
& a_{1}=\frac{2}{5} a+\frac{1}{5} b, \quad b_{1}=\frac{1}{5} a+\frac{2}{5} b, \\
& a_{2}=\frac{1}{25}(5 a+4 b), \quad b_{2}=\frac{1}{25}(4 a+5 b), \\
& a_{n}=\frac{1}{5^{n}}\left[\left(1+\frac{3^{n}-1}{2}\right) a+\frac{3^{n}-1}{2} b\right] \\
& b_{n}=\frac{1}{5^{n}}\left[\frac{3^{n}-1}{2} a+\left(1+\frac{3^{n}-1}{2}\right) b\right], \\
& c_{n}=\frac{2}{3} a_{n}+\frac{2}{3} b_{n} .
\end{aligned}
$$

For instance, the sequence $\left(b_{n}\right)$ describes the boundary decay of $h$ when approaching the boundary point $s_{3}$ (in the right corner of the Sierpinski gasket) from the left.

For horizontal lines, boundary value problems are studied in [30, 32, 36]. The harmonic function in the following example is computed in [30, Lemma 2.1]. Similar computations of harmonic functions have been done for other horizontal lines.

Example 4.3 Let $S$ be the Sierpinski gasket of side length 1. We impose zero boundary condition along the bottom line $L$ of the Sierpinski gasket, and boundary value 1 at the top vertex $s_{1}$ of the Sierpinski gasket, see Fig. 2. Let $U=S \backslash\left(L \cup\left\{s_{1}\right\}\right)$ be the remaining open domain in the Sierpinski gasket. Then there exists a unique non-negative continuous function $h$ on $S$ that is harmonic on $U$ and satisfies the given boundary condition. Moreover, the 


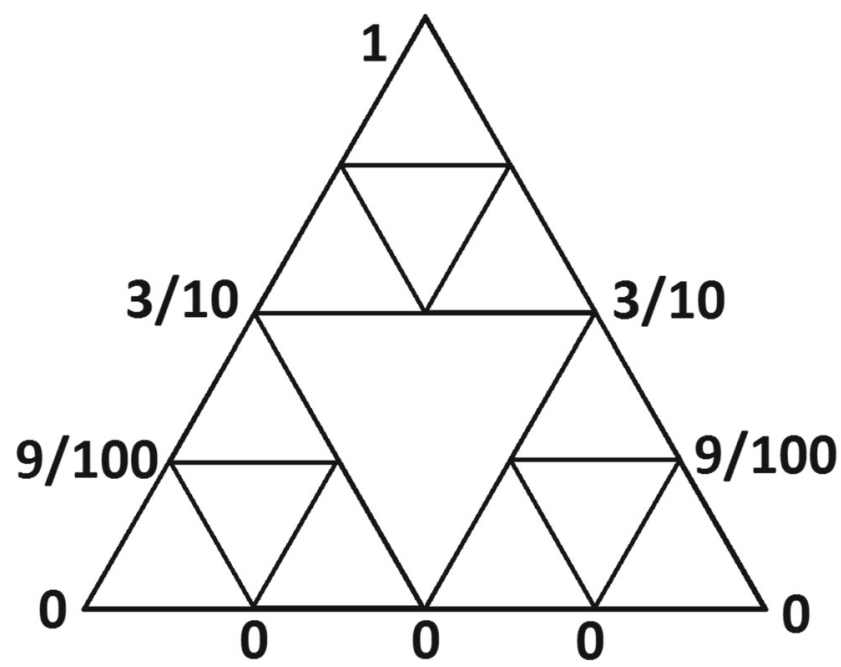

Fig. 2 Harmonic function on Sierpinski gasket with zero boundary condition on bottom line

Dirichlet heat kernel on $U$ has boundary decay rate $p_{U}^{D}(t, x, y) \asymp h(x) \asymp\left(\frac{3}{10}\right)^{\frac{\log d_{U}(x, L)}{\log 2}}$, and we have equality $h(x)=\left(\frac{3}{10}\right)^{n}$ whenever $d_{U}(x, L)=2^{-n}$.

Harmonic functions on domains to the left of a vertical line are studied in [37], as well as in [12].

\subsection{Sierpinski Carpet}

In the Sierpinski carpet, inner uniform domains can be constructed by the same token as in Proposition 4.1.

Proposition 4.4 Let L be a line in $\mathbb{R}^{2}$ that intersects the Sierpinski carpet. Let $U^{+}$and $U^{-}$ be the domains in the Sierpinski carpet that lie to either one side of the line. Let $U$ be either $U^{+}$or $U^{-}$. Then $U$ is an inner uniform domain in the Sierpinski carpet if and only if

(C') there exists a constant $c_{0} \in(0,1)$ such that for every square hole of side length $l$ in $U$, the distance between the line and the square hole is either 0 or greater than $c_{0} l$.

It is well known that the volume doubling property, the Poincare inequality and the cutoff Sobolev inequality are all satisfied by the Dirichlet form associated with the Laplacian on the Sierpinski carpet. Hence, if $U$ satisfies condition (C'), then Theorem 3.3 implies that the Dirichlet heat kernel exists on $U$ and satisfies the two-sided bounds provided by the theorem.

If condition (C') is not satisfied, then $U$ is a John domain and its center can be chosen among those of the four corners of the Sierpinski carpet that lie in $U$.

Acknowledgements A major part of this work was done while the author was affiliated with the University of Illinois at Urbana-Champaign. Discussions with Naotaka Kajino are gratefully acknowledged.

Funding Open Access funding enabled and organized by Projekt DEAL. 
Open Access This article is licensed under a Creative Commons Attribution 4.0 International License, which permits use, sharing, adaptation, distribution and reproduction in any medium or format, as long as you give appropriate credit to the original author(s) and the source, provide a link to the Creative Commons licence, and indicate if changes were made. The images or other third party material in this article are included in the article's Creative Commons licence, unless indicated otherwise in a credit line to the material. If material is not included in the article's Creative Commons licence and your intended use is not permitted by statutory regulation or exceeds the permitted use, you will need to obtain permission directly from the copyright holder. To view a copy of this licence, visit http://creativecommons.org/licenses/by/4.0/.

\section{References}

1. Aikawa, H.: Boundary Harnack principle and Martin boundary for a uniform domain. J. Math. Soc. Jpn. 53, 119-145 (2001)

2. Aikawa, H., Lundh, T., Mizutani, T.: Martin boundary of a fractal domain. Potential Anal. 18, 311-357 (2003)

3. Kigami, J.: Analysis on fractals, Cambridge Tracts in Mathematics. Cambridge University Press, pp. 143 (2001)

4. Andres, S., Barlow, M.T.: Energy inequalities for cutoff functions and some applications. J. Reine Angew. Math. 699, 183-215 (2015)

5. Bañuelos, R.: Intrinsic ultracontractivity and eigenfunction estimates for Schrödinger operators. J. Funct Anal. 100, 181-206 (1991)

6. Barlow, M.T., Perkins, E.A.: Brownian motion on the sierpinski gasket. Probab. Th. Rel. Fields 79 , 543-623 (1988)

7. Barlow, M.T., Bass, R.F.: Transition densities for Brownian motion on the Sierpiński carpet. Probab. Th. Rel. Fields 91, 307-330 (1992)

8. Martin, T.: Barlow and Richard F. bass, Brownian motion and harmonic analysis on Sierpinski carpets. Can. J. Math. 51(4), 673-744 (1999)

9. Barlow, M.T., Bass, R.F.: Stability of parabolic Harnack inequalities. Trans. Amer. Math. Soc. 356, 1501-1533 (2004)

10. Barlow, M.T., Bass, R.F., Kumagai, T.: Stability of parabolic Harnack inequalities on metric measure spaces. J. Math. Soc. Jpn. 58, 485-519 (2006)

11. Barlow, M.T., Grigor'yan, A., Kumagai, T.: On the equivalence of parabolic Harnack inequalities and heat kernel estimates. J. Math. Soc. Jpn. 64, 1091-1146 (2012)

12. Cao, S., Qiu, H.: Boundary Value Problems for harmonic functions on domains in Sierpinski gaskets. Commun. Pure Appl. Anal. 19(2), 1147-1179 (2020)

13. Chen, Z.-Q., Fukushima, M.: Symmetric Markov processes, time change, and boundary theory, London Mathematical Society Monographs Series, vol. 35, Princeton University Press, Princeton, pp. xvi+479 (2012)

14. Davies, E.B., Simon, B.: Ultracontractivity and the heat kernel for Schrödinger operators and Dirichlet Laplacians. J. Funct. Anal. 59, 335-395 (1984)

15. Eldredge, N., Saloff-Coste, L.: Widder's representation theorem for symmetric local Dirichlet spaces. J. Theoret. Probab. 27, 1178-1212 (2014)

16. Fitzsimmons, P.a.t.J., Hambly, B.M., Kumagai, T.: Transition density estimates for Brownian motion on affine nested fractals. Comm. Math. Phys. 165(3), 595-620 (1994)

17. Fukushima, M., Ōshima, Y., Takeda, M.: Dirichlet Forms and Symmetric Markov Processes, vol. 19 of De Gruyter Studies in Mathematics. Walter de Gruyter \& Co., Berlin (1994)

18. Grigor'yan, A., Hu, J., Lau, K.-S.: Generalized capacity, Harnack inequality and heat kernels of Dirichlet forms on metric measure spaces. J. Math. Soc. Jpn. 67, 1485-1549 (2015)

19. Grigor'yan, A., Telcs, A.: Two-sided estimates of heat kernels on metric measure spaces. Ann. Probab. 40(3), 1212-1284 (2012)

20. Gyrya, P., Saloff-Coste, L.: Neumann and Dirichlet heat kernels in inner uniform domains. Astérisque, pp. viii+144 (2011)

21. Hebisch, W., Saloff-Coste, L.: On the relation between elliptic and parabolic Harnack inequalities. Ann. Inst. Fourier (Grenoble) 51(5), 1437-1481 (2001)

22. Kajino, N.: Dirichlet heat kernel estimates in unbounded inner uniform domains of fractal-type spaces. In preparation

23. Kumagai, T.: Estimates of transition densities for Brownian motion on nested fractals. Probab. Theory Relat. Fields 96(2), 205-224 (1993) 
24. Lierl, J.: Parabolic Harnack inequality on fractal-type metric measure Dirichlet spaces. Rev. Mat. Iberoam. 2, 687-738 (2018)

25. Lierl, J.: Scale-invariant boundary Harnack principle on inner uniform domains in fractal-type spaces. Potential Anal. 43(4), 717-747 (2015)

26. Lierl, J.: Parabolic Harnack inequality for time-dependent non-symmetric Dirichlet forms. J. Math. Pures Appl. 140, 1-66 (2020)

27. Lierl, J., Saloff-Coste, L.: The Dirichlet heat kernel in inner uniform domains: Local results, compact domains and non-symmetric forms. J. Funct. Anal. 266, 4189-4235 (2014)

28. Lierl, J., Saloff-Coste, L.: Scale-invariant boundary Harnack principle in inner uniform domains. Osaka J. Math. 51, 619-656 (2014)

29. Lions, J.-L., Magenes, E.: Non-homogeneous boundary value problems and applications. vol. i, Springer, New York. Translated from the French by P. Kenneth, Die Grundlehren der mathematischen Wissenschaften, Band, pp. 181 (1972)

30. Owen, J., Strichartz, R.: Boundary value problems for harmonic functions on a domain in the Sierpinski gasket. Ind. Univ. Math. J. 1, 319-335 (2012)

31. Saloff-Coste, L.: Aspects of Sobolev-Type Inequalities, vol. 289 of London Mathematical Society Lecture Note Series. Cambridge University Press, Cambridge (2002)

32. Strichartz, R.: Some properties of Laplacians on fractals. J. Funct. Anal. 2, 181-208 (1999)

33. Sturm, K.-T.: Analysis on local Dirichlet spaces. III. The parabolic Harnack inequality. J. Math. Pures Appl. (9) 75, 273-297 (1996)

34. Willard, S.: General topology. Dover Publications, Inc., Mineola. Reprint of the 1970 original [AddisonWesley, Reading, MR0264581] (2004)

35. Wloka, J.: Partial differential equations. Cambridge University Press, Cambridge (1987). Translated from the German by C. B. Thomas and M. J. Thomas

36. Guo, Z., Kogan, R., Qiu, H., Strichartz, R.: Boundary value problems for a family of domains in the Sierpinski gasket. Illinois J. Math. 58(2), 497-519 (2014)

37. Li, W., Strichartz, R.: BOundary value problems on a half Sierpinski gasket. J. Fractal Geom. 1, 1-43 (2014)

Publisher's Note Springer Nature remains neutral with regard to jurisdictional claims in published maps and institutional affiliations. 